\title{
Asymptotic-Preserving methods and multiscale models for plasma physics
}

\author{
P. Degond ${ }^{\mathrm{a}}$, F. Deluzet ${ }^{\mathrm{b}, *}$ \\ ${ }^{a}$ Department of Mathematics, Imperial College London, \\ London SW7 2AZ, United Kingdom, \\ ${ }^{b}$ Université de Toulouse; UPS, INSA, UT1, UTM, Institut de Mathématiques de Toulouse, \\ CNRS, Institut de Mathématiques de Toulouse UMR 5219, F-31062 Toulouse, France,
}

\begin{abstract}
The purpose of the present paper is to provide an overview of AsymptoticPreserving methods for multiscale plasma simulations by addressing three singular perturbation problems. First, the quasi-neutral limit of fluid and kinetic models is investigated in the framework of non magnetized as well as magnetized plasmas. Second, the drift limit for fluid descriptions of thermal plasmas under large magnetic fields is addressed. Finally efficient numerical resolutions of anisotropic elliptic or diffusion equations arising in magnetized plasma simulation are reviewed.
\end{abstract}

Keywords: Asymptotic-Preserving method, Plasma, Quasi-neutrality, Drift limit, Anisotropic elliptic equations, Debye length, Singular limit

\section{Introduction}

Plasma physics is by essence a multiscale problem $[8,74,93]$ mixing microscopic to macroscopic scales. The microscopic space scales describe the motion of particles, their collisions on the mean free path scale, and the interaction with the electromagnetic fields over the plasma skin depth or the Debye length and the Larmor radius. The macroscopic scales are characteristic of the field and the plasma macroscopic evolution. The diversity in the time scales is also very wide. They range from high frequency phenomena defined by the propagation of electromagnetic waves at the speed of light as well as the cyclotron and the plasma frequencies; to the evolution of the coupled system composed of the plasma and the fields on the time scales of the plasma mean flow evolution. The simulation of these systems on large scales have been the source of an intense and fruitful research, with the derivation, analysis and implementation

${ }^{*}$ Corresponding author 
of models relating different description levels, from the microscopic scales to mesoscopic and macroscopic descriptions [17, 27, 28, 30, 103, 130].

Alongside, numerical methods have also been intensively developed with the same aim to produce simulations on large scales. Implicit methods [116, 35, 89, $97,107,144,32$ allow, to some extent, the de-correlation of the discretization parameters from the smallest scales described by the equations. The objective is thus to derive numerical methods with stability properties less restrictive than explicit methods. This gains the advantage of larger simulation parameters, chosen according to the scales of interest, rather than the small parameters described by the system.

However implicit discretizations may be ineffective when the stiffness of the problem leads to a degeneracy of the model. Fluid limits of kinetic equations are a good example of such frameworks. In the limit of a vanishing mean free path, the kinetic equation degenerates, constraining the distribution function to belong to the kernel of the collision operator. Unfortunately this only information does not permit to determine uniquely the distribution function. Therefore, the kinetic model becomes singular, while, in the limit of infinite collision rates, a fluid description is sufficient. A similar difficulty occurs for the diffusion equation derived in Sec. 3 as illustrated by the investigations of Sec. 4 addressing the associated steady state problem. This issue is usually overcome thanks to domain decomposition strategies, consisting in using $P^{0}$ the asymptotic (macroscopic) description everywhere $P^{\varepsilon}$ the multiscale (microscopic) model is not compulsory. However, this coupling strategy is still an open question, at least in specific contexts (see for instance [99, 125] and the references therein for examples of domain decomposition). The main difficulty raised by this strategy is twofold. First, it is not necessarily straightforward to match the unknowns advanced by the multiscale model with those of the limit one [99]. Second, the interface delimiting the sub-domains may be evolving with the system, its location computation being a problem by its own. On top of that, this interface should be immersed in a part of the domain where both the multiscale and the limit models are valid. The existence of such a region supposes that the approximation error of the numerical method dominates the modelling error produced by the use of the limit model for non vanishing asymptotic parameter values. For refined numerical parameters this requirement may not be met (an example of such a situation is discussed in [39]), or at the price of important computational efforts.

An alternative approach has been introduced in the frame of AsymptoticPreserving methods originally designed to cope with fluid and diffusive limits of kinetic equations [98]. The purpose is to derive numerical methods bridging the microscopic description and the asymptotic model when this latter is derived as a singular limit of the former model. Fluid and diffusive limits of kinetic equations remain the most active field of developments for AP schemes outlined by numerous publications $[58,124,9,111,45,125,38,67,120,67,19,26,96$, $95,43,104]$. Other singular limits are however addressed, among which the low Mach regime for fluid systems [60, 63, 82, 37, 140, 122], with applications to semiconductors [68, 13], nano-structures and quantum systems [59, 10, 46, 92] 
this list being non exhaustive. We also refer to $[99,48]$ for reviews on AP methods. Note also related works on relaxation limit of hyperbolic systems [100], radiative transfer [109, 108] and low Mach regimes [85, 3, 36] sharing analogies with AP-methods.

Asymptotic-Preserving numerical methods are constructed to offer a consistent discretization of the multiscale model $\left(P^{\varepsilon}\right)$ when the discretization parameters resolve the small scales. Conversely, for vanishing $\varepsilon$, AP-methods are consistent with the limit model $\left(P^{0}\right)$, verified by the limit of the solution of $\left(P^{\varepsilon}\right)$. This is represented by the celebrated diagram depicted on Fig. 1. In addition to these consistency properties, AP-schemes have stability properties independent of $\varepsilon$.
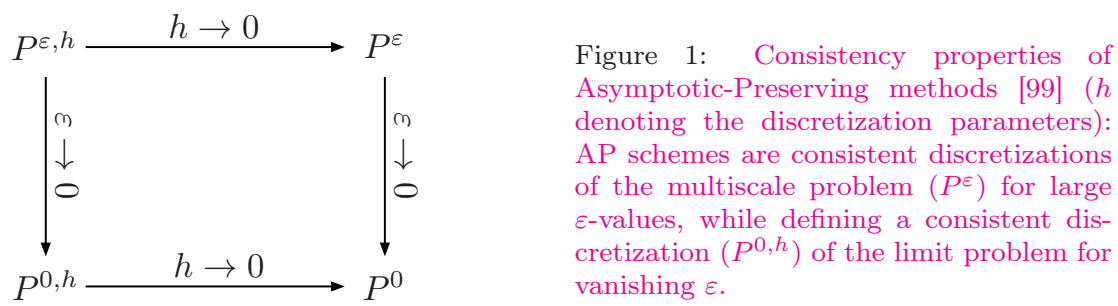

In this document AP-Schemes are constructed as discretizations of a reformulated system: a set of equations equivalent to the multiscale problem but, in which, the limit $\varepsilon \rightarrow 0$ is regular. This differs from classical derivations of implicit discretizations. Indeed, AP-methods are constructed to bridge several sets of equations describing the system in different regimes, rather than alleviating the stability constraints of the numerical methods. The aim here is to shape a single set of reformulated equations unifying these regimes. In this reformulated system the macroscopic problem is recovered for vanishing asymptotic parameters, hence the regular nature of the limit $\varepsilon \rightarrow 0$ in this set of equations. Three main steps can be identified in the derivation of these methods. The first one consists in elaborating the limit problem. This system is defined as the set of well posed equations providing the limit of the solution in the asymptotic regime. In the framework of singular perturbation problems, the limit problem is not readily obtained by setting the asymptotic parameter to zero in the multiscale problem. The second step consists in deriving the reformulated set of equations mentioned above. In this aim a good understanding of the derivation of the limit problem is mandatory. Finally the time discretization is designed to meet consistency requirements with all regimes. This necessitates a sufficient implicitation level in order to guarantee the consistency with the different regimes, providing thus a means of computing all the unknowns whatever the values of the asymptotic parameter.

The purpose of the present paper is to illustrate these concepts thanks to three singular perturbation problems.

The first one is related to the quasi-neutral limit of fluid and kinetic plasma models coupled to the Maxwell system. In many applications the charge sep- 
arations can be disregarded at the scales of interest, the plasma being thus considered as quasi-neutral. This property is harnessed to derive reduced models, filtering the scales describing the charge separations out from the equations. However, an accurate description of the whole system on large scales generally requires the resolution of the complex physics occurring in limited regions, for instance near the plasma boundary or, to describe the interaction of the plasma with a wall (see $[106,126,137])$ where electrostatic sheathes may appear. In these regions the assumptions used to derive quasi-neutral models are not valid. More complex models are needed to account for the physics prevailing in these regions, calling for numerical methods able to treat efficiently quasi-neutral models with local break down. The difficulty of this asymptotic is explained by the degeneracy of the equation providing the electric field. In non quasi-neutral models, the electric field is computed from the Maxwell system [14, 91, 138]. In the quasi-neutral regime, these equations being degenerate, the electric field is computed from the equations describing the evolution of the electrons and the ions. These regimes translate thus two different physical phenomena, accounting for the singular nature of this limit addressed in Sec. 2.

The second context is specific to hot plasmas evolving in the presence of strong magnetic fields. These investigations are limited to fluid descriptions with an external magnetic field. The purpose here, is to address the so called gyro-fluid regime [83] which bears some analogies with drift approximations $[15,103,135,130,79,139,127,110]$ largely used for the modelling of tokamak plasmas on large time scales. The drift regime is characterized by a vanishing inertia, which is responsible of the momentum equation degeneracy. Along the magnetic field lines, this equation imposes a zero force regime with the pressure gradient balancing the electric force. No explicit occurrence of the parallel momentum survives in the drift asymptotic which explains the singularity of this limit. A regimes transition is observed near the wall, with a significant decrease of the plasma temperature and more importantly an acceleration of the particles by the electrostatic field existing in the sheath $([33,137])$. In this area the particle inertia becomes again significant breaking the drift assumption. The derivation of asymptotic preserving methods in this framework is detailed in Sec. 3.

Finally, we propose a review of AP-methods for anisotropic elliptic or diffusion equations arising in the simulation of tokamak [44] and ionospheric [12] plasmas. In a plasma under large magnetic fields, the diffusion along the magnetic field lines is almost infinite in the time scale of the dynamic in the perpendicular directions $[81,134,64,73]$. The difficulty raised by this anisotropy in the context of tokamaks is due to the periodicity of the torus. Indeed, in the limit of an infinite diffusion in the aligned direction, the parallel diffusion is dominant, this operator being supplemented with periodic boundary conditions at each ends of the magnetic field lines. Therefore, its kernel is not reduced to zero, leading to a difficulty comparable to the one referred above concerning the limit of infinite collisions for kinetic descriptions. This issue is illustrated thanks to a simplified problem in the beginning of Sec. 4 and investigated by means of AP-methods in the sequel of the section. 


\section{Quasi-neutral limit of kinetic and fluid plasma descriptions}

\subsection{Introduction}

The quasi-neutral limit can be related to the so-called plasma approximation as defined in [30] which consists, for dense plasmas, in assuming equal ionic and electronic densities $n_{i}=n_{e}$ together with an electric field that is not divergence free $\nabla \cdot E \neq 0$. This might appear as a paradox since it breaks the Gauss equation $\nabla \cdot E=q\left(n_{i}-n_{e}\right) / \varepsilon_{0}$ ( $\varepsilon_{0}$ being the vacuum permittivity and $q$ the elementary charge). The full set of equations defining the Maxwell system writes

$$
\begin{aligned}
& \frac{1}{c^{2}} \frac{\partial E}{\partial t}-\nabla \times B=-\mu_{0} J, \\
& \frac{\partial B}{\partial t}+\nabla \times E=0, \\
& \nabla \cdot E=\frac{\rho}{\varepsilon_{0}}, \\
& \nabla \cdot B=0 .
\end{aligned}
$$

It consists of the Ampère (1a), the Faraday (1b), the Gauss (1c) and the Maxwell-Thomson (1d) equations. In this system $(E, B)$ is the electromagnetic field, $c$ the speed of light, $\mu_{0}$ and $\varepsilon_{0}$ being the vacuum permeability and permittivity, with $\varepsilon_{0} \mu_{0} c^{2}=1$. The sources of Maxwell's equations, namely the charge and current densities $(\rho, J)$, are defined by the electronic and ionic densities and mean velocities as $\rho=q\left(n_{i}-n_{e}\right)$ and $J=q\left(n_{i} u_{i}-n_{e} u_{e}\right)$. They verify the continuity equation

$$
\frac{\partial \rho}{\partial t}+\nabla \cdot J=0
$$

Note that computing formally the divergence of Eq. (1a), owing to the continuity equation (2), the time derivative of Eq. (1c) is recovered. This means that the Gauss equation is verified for $t>0$ if it holds true at initial time. The same remark applies for the Maxwell-Thompson equation (1d) which is a consequence of Eq. (1b). The only Ampère and Faraday equations are thus sufficient to advance the electromagnetic field.

The plasma approximation ambiguity is clarified in Sec. 2.2 thanks to the analysis of the different orderings revealed by a scaling of the Maxwell system. The inter-relations of the dimensionless parameters occurring in this set of rescaled equations define different regimes. The first one relates the propagation of an electromagnetic wave at the speed of light and is referred to as the Maxwell regime. In this ordering, the Maxwell sources vanish and do not contribute to the changes in the electromagnetic field, the electric field being computed by means of the displacement $\left(1 / c^{2}\right)(\partial E / \partial t)$ current in Eq. (1a). On the contrary, the evolution in the quasi-neutral regime is dominated by the sources with a negligible displacement current. Therefore, the transitions between the Maxwell and the quasi-neutral regimes rely on the relative influence of the displacement current $\left(1 / c^{2}\right)(\partial E / \partial t)$ and the current of particles $J$. The vanishing of the 
displacement current in the Ampère equation is at the origin of the singular nature of the quasi-neutral limit. Finally the electrostatic limit of the Maxwell system is discussed. It defines the electric field for vanishing magnetic fields, the Maxwell system thus reducing to the only Gauss equation (1c). The aim is to characterize the quasi-neutral limit in this regime. In particular, the singular nature of the quasi-neutral limit is illustrated also in this framework, with the degeneracy of the Gauss equation.

In the quasi-neutral asymptotic, the degeneracy of the Ampère and the Gauss equations call for new means of computing the electric field. This is classically achieved thanks to a generalized Ohm law. Ohm's law is derived from the equations describing the plasma evolution thanks to either the quasineutral constraint $\nabla \cdot J=0$ or, the electronic momentum conservation, in which the inertia is neglected. In its simplest form it reduces to $E=-u_{e} \times B, u_{e}$ being the electron velocity. We refer to [106] for some seminal works on quasi-neutral models, to [88] for a short review, [148, 102, 149, 40, 42, 143, 142] and the references therein for implementations of quasi-neutral plasma models.

However quasi-neutral descriptions have a limited range of validity. In particular these models are not valid in vacuum or low plasma density regions where high frequency phenomena may occur [141]. The purpose of the AsymptoticPreserving methods reviewed in this paper is to bring the two regimes into a single set of equations, making possible the transition between the evolution of the electric field in the Maxwell regime, by means of the displacement current and, that of the quasi-neutral regime, with an electric field computed thanks to a generalized Ohm law. In this respect, AP methods implement the guide line stated in [30] "do not use Maxwell's equations to compute the electric field unless it is unavoidable?".

The derivation of the AP methods is addressed in Sec. 2.3. One key point is to identify the equations describing the system in the quasi-neutral regime. The Ohm's law heavily relying on the equations describing the particles evolution, the plasma models, either fluid or kinetic, are therefore an important aspect to consider in designing AP methods. In Sec. 2.3 a unified presentation of the different regimes is proposed by means of the "augmented" Vlasov-Maxwell system. This choice offers different advantages. First, the augmented system contains the difficulty of both the electromagnetic and the electrostatic regimes. Second, the Ohm law being derived from equations driving the evolution of macroscopic quantities, the construction of AP methods can be readily transposed from the kinetic to the fluid framework. Finally an overview of the numerical implementations of AP-methods is proposed in Sec. 2.3.2.

\subsection{Outlines of the quasi-neutral and electrostatic limits of the Maxwell system}

The objectives of this section are twofold. On the one hand, the quasi-neutral limit is investigated thanks to a scaling of the equations in order to explain the paradox raised in the introduction. On the other hand, some consistency properties, relating the Ampère and the Gauss equations are outlined. The electrostatic limit of the Maxwell system is also discussed. 
The model is first stated thanks to dimensionless variables. The aim of this procedure is to highlight the stiff terms in the equations thanks to the introduction of dimensionless parameters. The physical variables are scaled by their typical value, $\bar{x}$ and $\bar{t}$ being the space and time scales, the following identity holds $x=\bar{x} x^{\prime}$ and $t=\bar{t} t^{\prime}, x^{\prime}$ and $t^{\prime}$ denoting the dimensionless variables. These scales define $\bar{\vartheta}=\bar{x} / \bar{t}$ the reference velocity, it is related the velocity characterizing the changes in the electromagnetic field. The typical magnitude of the electric and magnetic fields are denoted $\bar{E}$ and $\bar{B}$. The plasma characteristics are denoted $\bar{T}, \bar{u}$ and $\bar{n}$ for the typical temperature, mean velocity and density, allowing the definition of the electronic thermal velocity and plasma frequency

$$
v_{t h, e}=\left(\frac{k_{B} \bar{T}}{m_{e}}\right)^{1 / 2}, \quad \omega_{p, e}=\left(\frac{q^{2} \bar{n}}{\varepsilon_{0} m_{e}}\right)^{1 / 2}
$$

with $m_{e}$ the electronic mass, and the Debye length

$$
\lambda_{D}=\frac{v_{t h, e}}{\omega_{p, e}}=\left(\frac{\varepsilon_{0} k_{B} \bar{T}}{q^{2} \bar{n}}\right)^{1 / 2},
$$

$k_{B}$ denoting the Boltzmann constant. The Maxwell sources are scaled with $\bar{\rho}=q \bar{n}$ and $\bar{J}=q \bar{n} \bar{u}$ for the charge and current densities. The introduction of the re-scaled variables into the equations reveals some dimensionless parameters:

$$
\left\{\begin{array}{l}
\alpha=\frac{\bar{\vartheta}}{c}, \text { the reference velocity to the speed of light; } \\
\zeta=\frac{\bar{u}}{\bar{\vartheta}}, \text { the plasma mean velocity relative to the speed of interest; } \\
M=\frac{\bar{u}}{v_{t h, e}} \text { the electronic Mach number; } \\
\eta=\frac{q \bar{E} \bar{x}}{m_{e} \bar{u}^{2}}, \text { the ratio of the electric and plasma kinetic energies; } \\
\beta=\frac{\bar{\vartheta} \bar{B}}{\bar{E}}, \text { the induced electric field to the total electric field } \\
\lambda=\frac{\lambda_{D}}{\bar{x}}, \text { the dimensionless Debye length. }
\end{array}\right.
$$

With these dimensionless parameters, the scaled system is recast into

$$
\begin{aligned}
& \lambda^{2} \frac{\partial E}{\partial t}-\beta \frac{\lambda^{2}}{\alpha^{2}} \nabla \times B=-\frac{\zeta}{\eta M^{2}} J, \\
& \beta \frac{\partial B}{\partial t}+\nabla \times E=0, \\
& \lambda^{2} \eta M^{2} \nabla \cdot E=\rho, \\
& \nabla \cdot B=0, \\
& \frac{\partial \rho}{\partial t}+\zeta \nabla \cdot J=0,
\end{aligned}
$$


where, for sake of readability, the primes are omitted for the scaled variables.

Two regimes can be identified accordingly to the frequency range characterizing the system evolution, which is related to the assumptions made on the reference velocity In the high frequency limit, referred to as the Maxwell regime in the sequel, the reference velocity is assumed comparable to the speed of light and large compared to both the mean velocity of the plasma and the particles thermal velocity. The Debye length is assumed to be large compared to the typical space scale which amounts to consider low plasma densities, the other parameter being assumed to be the same order of magnitude. This translates into the following scaling relations

$$
\zeta \ll 1, \quad \alpha^{2} \sim \beta \sim \eta \sim M^{2} \sim 1, \quad \lambda^{2} \gg 1 .
$$

The system reduces to the homogeneous Maxwell equations with the propagation of the electromagnetic wave at the speed of light with an electromagnetic field non affected by the plasma evolution

$$
\begin{aligned}
& \frac{\partial E}{\partial t}-\nabla \times B=0, \\
& \frac{\partial B}{\partial t}+\nabla \times E=0 \\
& \nabla \cdot E=0, \\
& \nabla \cdot B=0,
\end{aligned}
$$

The electric field is computed by means of the displacement current in (M-A).

The quasi-neutral limit is defined by a reference velocity comparable to the plasma mean velocity and the particles thermal velocity, these velocities being assumed small compared to the speed of light. The regime is therefore a low frequency asymptotic. The scaled Debye length is also assumed to define a small scale in this regime which finally yields to the scaling relations

$$
\lambda^{2} \sim \alpha^{2} \ll 1, \quad \zeta \sim \beta \sim \eta \sim M^{2} \sim 1 .
$$

The scaling relation $\bar{E} \sim \bar{u} \bar{B}(\beta \sim 1, \zeta \sim 1)$ is common to all Magneto-HydroDynamic (MHD) models and referred to as the frozen field assumption. It translates that, in a dense plasma, the magnetic field is convected with the plasma flow at the typical velocity is $\bar{u}$. In particular, the propagation of electromagnetic waves at the speed of light is not possible in a dense plasma $[103,30,17,74]$ and therefore not described by quasi-neutral models. The other dimensionless parameters may be assumed of order one. In the quasi-neutral limit $(\alpha, \lambda) \rightarrow 0$, the equations write

$$
\begin{aligned}
& \nabla \times B=J, \\
& \frac{\partial B}{\partial t}+\nabla \times E=0 \\
& 0=\rho, \\
& \nabla \cdot B=0,
\end{aligned}
$$


the continuity equation providing $\nabla \cdot J=0$. The Gauss equation (M-G) provides the quasi-neutral assumption $\rho=0$, the electric field contribution vanishing in this equation. Therefore, this allows for the computation of non divergence free electric field and explains the paradox raised by a crude review of the dimensional equations. The Ampère equation also degenerates in the asymptotic $\lambda^{2} \rightarrow 0$ along with $\alpha^{2} / \lambda^{2}=\mathcal{O}(1)$, with a vanishing displacement current. In this equation too, the occurrence of the electric field vanishes in the quasi-neutral limit which leads to the conclusion that the (homogeneous) Maxwell equations cannot be used to compute the electric field. More precisely, the electric field must be found from $J[30]$ by means of a generalized Ohm's law, explaining how the current of particles $J$ and the electric field relate to each other. This is routinely implemented in quasi-neutral descriptions of plasmas, the most widely used being the Magneto-Hydro-Dynamic (MHD) models [15, 47, 76, 135].

The Maxwell system may be simplified when the magnetic component of the field is a constant or vanishes, the regime being referred to as electrostatic. The quasi-neutral limits of electrostatic plasma descriptions are also singular. This features is highlighted in the next lines. In this aim, the electrostatic limit of the Maxwell system is first derived by letting $\alpha \rightarrow 0$. Indeed, by the equation (M-A) the magnetic field is curl free $(\nabla \times B=0)$, which together with $\nabla \cdot B=0$ and assuming adequate boundary conditions, provides a constant magnetic field. The equation (M-F) provides thus a curl free electric field $\nabla \times E=0$ assumed to derive from a potential $E=-\nabla \phi$. In this regime, the Ampère equation can be decomposed into a curl free and a divergence free identity

$$
\begin{aligned}
\lambda^{2} \frac{\partial}{\partial t} \nabla \phi & =\frac{\zeta}{\eta M^{2}} J_{L}, \\
\beta \frac{\lambda^{2}}{\alpha^{2}} \nabla \times B & =\frac{\zeta}{\eta M^{2}} J_{T},
\end{aligned}
$$

where $J=J_{L}+J_{T}, \nabla \cdot J_{T}=0$ and $\nabla \times J_{L}=0^{1}$. While $\nabla \times B$ vanishes in the electrostatic limit $\alpha \rightarrow 0$, the quantity $\nabla \times B / \alpha^{2}$ remains finite as long as the current transverse part $J_{T}$ does not vanish. However it does not contribute to the definition of the electrostatic field, as outlined by the decomposition (8). This feature can also be recovered by computing formally the divergence of the Ampère equation (M-A), providing

$$
\lambda^{2} \frac{\partial}{\partial t} \Delta \phi=\frac{\zeta}{\eta M^{2}} \nabla \cdot J .
$$

This equation together with the continuity equation $(\mathrm{C})$ provides

$$
-\lambda^{2} \eta M^{2} \frac{\partial}{\partial t} \Delta \phi=\frac{\partial \rho}{\partial t} .
$$

\footnotetext{
${ }^{1}$ Performing a Fourier transform of these identities yields $k \cdot J_{T}=0$ and $k \times J_{L}=0$, with $k$ the wave vector associated to $x$, hence the terminology "Transverse" and "Longitudinal" with respect to $k$.
} 
This outlines that the Gauss equation is a consequence of the Ampère (M-A) and the continuity $(\mathrm{C})$ equations. It remains verified if it holds true at initial time. Consequently, in the electrostatic regime, the only Gauss equation is sufficient to compute the entire electric field, this equation being usually substituted to the whole Maxwell system to give rise to the so-called Poisson equation

$$
-\lambda^{2} \eta M^{2} \Delta \phi=\rho .
$$

Note that, the quasi-neutral limit is thus defined by $\lambda^{2} \rightarrow 0$ together with $\lambda^{2} / \alpha^{2} \rightarrow 0$, in contrast to $\lambda^{2} \rightarrow 0$ and $\lambda^{2} / \alpha^{2}=\mathcal{O}(1)$ for the electromagnetic framework.

\subsection{Asymptotic-Preserving formulation of the Vlasov-Maxwell system}

\subsubsection{The scaled Vlasov-Maxwell system}

The model investigated here consists of the Maxwell system (1) coupled to a Vlasov equation for the electrons, the ions being assumed at rest with a uniform density to simplify the notations. The distribution function, denoted $f$, depends on $x \in \Omega_{x} \subset \mathbb{R}^{3}$, the microscopic velocity $v \in \Omega_{v} \subset \mathbb{R}^{3}$ and on time $t \in \mathbb{R}^{+}$. The function is the solution to

$$
\frac{\partial f}{\partial t}+v \cdot \nabla f-\frac{q}{m_{e}}(E+v \times B) \cdot \nabla_{v} f=0 .
$$

In order to address straightforwardly the asymptotic regime, the scaling defined by Eq.(5) is again harnessed, but to simplify, with $\bar{u}=\bar{\vartheta}$, where $\bar{\vartheta}=\bar{x} / \bar{t}$ and $\bar{u}$ is the mean plasma velocity, which amounts to setting $\zeta=1$. The particles velocity $v$ being scaled with the electronic thermal velocity (3). In the sequel similar scaling relations as the ones defining the quasi-neutral regime (7) will be considered. To simplify further the writing, the two small scales $\alpha$ and $\lambda$ will be denoted by a single parameter $\lambda$, so that the quasi-neutral regime is easily identified by the limit $\lambda \rightarrow 0$. The dimensionless Vlasov-Maxwell system is

$$
(V M)^{\lambda}\left\{\begin{array}{l}
\frac{\partial f}{\partial t}+v \cdot \nabla f-(E+v \times B) \cdot \nabla_{v} f=0 \\
\lambda^{2} \frac{\partial E}{\partial t}-\nabla \times B=-J \\
\frac{\partial B}{\partial t}+\nabla \times E=0 \\
\lambda^{2} \nabla \cdot E=1-n \\
\nabla \cdot B=0
\end{array}\right.
$$

with

$$
n=\int_{\Omega_{v}} f(x, v, t) d v, \quad J=-\int_{\Omega_{v}} f(x, v, t) v d v
$$


The sources of the Maxwell system verify a continuity equation derived from the moments of the Vlasov equation (11a) giving rise to

$$
\begin{aligned}
& \frac{\partial n}{\partial t}-\nabla \cdot J=0, \\
& \frac{\partial J}{\partial t}-\nabla \cdot \mathbb{S}=(n E-J \times B), \quad \mathbb{S}=\int_{\Omega_{v}} f(x, v, t) v \otimes v d v .
\end{aligned}
$$

As outlined in section 2.2, the Gauss equation is a consequence of the Ampère equation (11b) and the continuity equation (12a). However, the consistency with this latter is not always satisfied by numerical methods. This is for instance a common flaw of Particle-In-Cell methods largely documented (see for instance $[14,5]$ ). The most widely adopted solution is the correction of the electric field predicted by the Ampère equation. This correction is computed by an electrostatic potential $p$ verifying the Gauss equation (11d). This is the so-called Boris correction [18] decomposed in two steps. First the predicted electric field $\tilde{E}$ is computed by means of the Ampère equation. Second the correction is applied to this field, defining the corrected field $E=\tilde{E}-\nabla p$ in order for the Gauss equation to be satisfied:

$$
\lambda^{2} \nabla \cdot E=1-n, \quad E=\tilde{E}-\nabla p,
$$

This gives rise to the dimensionless Vlasov-Maxwell system augmented with the corrector $p$

$$
(a V M)^{\lambda}\left\{\begin{array}{l}
\frac{\partial f}{\partial t}+v \cdot \nabla f-(E+v \times B) \cdot \nabla_{v} f=0 \\
\lambda^{2} \frac{\partial \tilde{E}}{\partial t}-\nabla \times B=-J \\
\frac{\partial B}{\partial t}+\nabla \times \tilde{E}=0 \\
\lambda^{2} \Delta p=\lambda^{2} \nabla \cdot \tilde{E}-(1-n), \\
\nabla \cdot B=0 \\
E=\tilde{E}-\nabla p .
\end{array}\right.
$$

In this system, the corrected field is used to advance the distribution function. Note that either $E$ or $\tilde{E}$ may be used in equation (14c) owing to the property $\nabla \times \nabla p=0$. The right hand side of Eq. (14d) can be interpreted as the consistency default with the Gauss equation. The corrector $p$ vanishes, subject to the boundary conditions, if the electric field carried out by the Ampère equation verifies the Gauss equation.

The difficulty in handling the quasi-neutral limit is thus twofold. In addition to the degeneracy of the Ampère equation (14b), a means of computing the corrector needs to be worked out for the limit regime, the Gauss equation (14d) also degenerating in the quasi-neutral limit. This last difficulty is similar to the one posed by the computation of the electric potential in the electrostatic 
framework. The investigation of the augmented Vlasov-Maxwell system is thus a good means of offering a unified presentation of the quasi-neutral limit for both the electrostatic and electromagnetic regimes.

\subsubsection{Quasi-neutral model and reformulation of the augmented Vlasov-Maxwell system}

The objective here is to restore a means of computing the electric field in the quasi-neutral regime. As mentioned above, in the Maxwell regime, the electric field is computed thanks to the displacement current. The displacement current being multiplied by $\lambda^{2}$ it is dropped out of the Ampère equation in the quasineutral limit. Therefore a Ohm law needs to be considered in order to express how the electric field relates to $J$ the current of particles. This finally restores a means of computing the electric field in the Ampère equation. Letting $\lambda^{2} \rightarrow 0$ in (14b) and taking the formal time derivative of this equation together with the curl of Faraday's law (14c) yields

$$
\nabla \times \nabla \times E=\frac{\partial J}{\partial t}
$$

In this equation a link between the electric field and the electric sources is restored. However, it does not allow for the computation of the entire electric field. Indeed, the solution of this equation can be augmented by any gradient without changing the equality: the electrostatic component of the field cannot be uniquely determined from (15). This is corrected thanks to the expression of the current of particles which translates the response of the particles to the electric field, with

$$
\frac{\partial J}{\partial t}=\nabla \cdot \mathbb{S}+n \tilde{E}-J \times B
$$

Inserting this definition into (15), the quasi-neutral equation providing the entire electric field in the quasi-neutral regime can be precised, with

$$
\nabla \times \nabla \times \tilde{E}+n \tilde{E}=J \times B-\nabla \cdot \mathbb{S} .
$$

A similar reformulation can be performed for the correction potential $p$. Indeed, Eq. (14d) degenerates into the quasi-neutrality relation $1-n=0$. This constraint is operated together with the moments of the Vlasov equation in order to derive the equation verified by the corrector. Following the spirit of the Boris procedure, a correction of the electric field is introduced in order for the continuity equation to be verified. Taking the double time derivative of this equation together with the moments of the Vlasov equation, in which the electric field is corrected, the following equation is derived

$$
\frac{\partial^{2} n}{\partial t^{2}}=\nabla \cdot \frac{\partial J}{\partial t}=\nabla^{2}: \mathbb{S}+\nabla \cdot(n(\tilde{E}-\nabla p))-\nabla \cdot(J \times B),
$$

where $\nabla^{2}: \mathbb{S}:=\nabla \cdot(\nabla \cdot \mathbb{S})$. This finally provides the equation verified by the corrector in the limit $\lambda^{2} \rightarrow 0$, so that it is possible to state the quasi-neutral 
Vlasov-Maxwell system

$$
(a V M)^{0}\left\{\begin{array}{l}
\frac{\partial f}{\partial t}+v \cdot \nabla f-(E+v \times B) \cdot \nabla_{v} f=0 \\
\nabla \times \nabla \times \tilde{E}+n \tilde{E}=J \times B-\nabla \cdot \mathbb{S}, \\
\frac{\partial B}{\partial t}+\nabla \times \tilde{E}=0, \\
-\nabla \cdot(n \nabla p)=\frac{\partial^{2} n}{\partial t^{2}}-\nabla^{2}: \mathbb{S}-\nabla \cdot(n \tilde{E})+\nabla \cdot(J \times B), \\
\nabla \cdot B=0, \\
E=\tilde{E}-\nabla p .
\end{array}\right.
$$

In the quasi-neutral limit, the electric field can be interpreted as the Lagrange multiplier of the equilibrium $\nabla \times B=J$, the corrector potential as the Lagrange multiplier of the constraint $\nabla \cdot J=0$, or, more precisely to the time derivative of these identities. The equation (17b) outlines the singular nature of the quasineutral limit: the electric field verifies a hyperbolic equation in the Maxwell regime defined in section 2.1 while it is computed thanks to an elliptic equation in the quasi-neutral limit. On top of that, these two equations relate different physical phenomena, the propagation of an electromagnetic wave at the speed of light on the one hand, the response of the charged particles to the electric field on the other hand. The quasi-neutral regime investigated with this limit model is close to a kinetic description of the so-called Electron MHD [78] and the quasi-neutral model identified in [142]. In this system the scale of interest is that of the electron, rather than the ion dynamics in the classical MHD models, with a finite electron inertia. Moreover the model defined by (17) remains a fully kinetic description for the plasma.

The aim of the reformulation, leading to an asymptotic preserving method, is to bring these two regimes into a single set of equations with a smooth transition from one to the other one according to the values of $\lambda$. With this aim, a derivation similar to that of the limit problem (17) is performed but keeping $\lambda>0$. This yields the reformulated Vlasov-Maxwell system

$$
(R a V M)^{\lambda}\left\{\begin{array}{l}
\frac{\partial f}{\partial t}+v \cdot \nabla f-(E+v \times B) \cdot \nabla_{v} f=0 \\
\lambda^{2} \frac{\partial^{2} \tilde{E}}{\partial t^{2}}+\nabla \times \nabla \times \tilde{E}+n \tilde{E}=J \times B-\nabla \cdot \mathbb{S}, \\
\frac{\partial B}{\partial t}+\nabla \times \tilde{E}=0, \\
-\lambda^{2} \frac{\partial^{2}}{\partial t^{2}} \Delta p-\nabla \cdot(n \nabla p)= \\
\quad \frac{\partial^{2} n}{\partial t^{2}}-\nabla^{2}: \mathbb{S}-\nabla \cdot(n \tilde{E})+\nabla \cdot(J \times B), \\
\nabla \cdot B=0, \\
E=\tilde{E}-\nabla p .
\end{array}\right.
$$


Remark 2.1. a) The reformulated Ampère equation (18b) is well posed (provided adequate boundary conditions) for all values of $\lambda^{2}$. Indeed in the limit $\lambda \rightarrow 0$ the plasma density is large and the operator $\nabla \times \nabla \times E+n E$ is elliptic. Conversely, when $n \rightarrow 0$ the scaled Debye length is large and the equation remains well posed. These remarks also apply to the reformulated Gauss equation (18d) providing the corrector.

b) The quasi-neutral Vlasov-Maxwell system (17) is recovered from the reformulated system when $\lambda \rightarrow 0$. The quasi-neutral limit is a regular perturbation of the reformulated system (18).

c) The right hand side of the equation (18d) can be interpreted as the default of consistency with the continuity equation a common feature with the Boris correction [18]. In this respect, this equation can be regarded as a generalization of the Boris correction.

\subsection{Overview of the numerical methods}

\subsubsection{Asymptotic-Preserving time discretization}

The purpose here is to use the concepts introduced in the precedent section for the continuous system and to transpose them to the discrete equations. Generally, the time discretization is a key point in the derivation of an AP numerical method. Due to the singular nature of the quasi-neutral limit several quantities must be computed thanks to an implicit time discretization in order to secure the consistency with both the Maxwell and the quasi-neutral regimes and to provide a means of computing the electric field in every regime.

The level of implicitness is controlled by three parameters $(a, b, c)$, the value of each one being equal to either 1 or 0 and commented in the following lines.

$$
\begin{aligned}
& \frac{1}{\Delta t}\left(n^{m+1}-n^{m}\right)-\nabla \cdot J^{m+a}=0, \\
& \frac{1}{\Delta t}\left(J^{m+1}-J^{m}\right)-\nabla \cdot \mathbb{S}^{m}=n^{m+1-a} E^{m+1}-J^{m} \times B^{m}, \\
& \frac{1}{\Delta t}\left(B^{m+1}-B^{m}\right)+\nabla \times E^{m+b}=0, \\
& \lambda^{2} \frac{1}{\Delta t}\left(\tilde{E}^{m+1}-E^{m}\right)-\nabla \times B^{m+c}=-J^{m+a}, \\
& \lambda^{2} \nabla \cdot E^{m+1}=\left(1-n^{m+1}\right), \\
& E^{m+1}=\tilde{E}^{m+1}-\nabla p .
\end{aligned}
$$

supplemented with $\nabla \cdot B^{m+1}=0$.

At this stage, different remarks can be stated:

a) The quasi-neutral regime is recovered for vanishing $\lambda$ which stands for both

the scaled Debye length and the ratio of the reference velocity to the speed of 
light. The stability with respect to $\lambda$ requires therefore an implicit discretization of the (homogeneous) Maxwell equations, yielding to $b=c=1$. This is related to the assumption that the reference velocity is small compared to the speed of light $(\alpha \rightarrow 0)$.

b) The consistency property with respect to the quasi-neutral regime requires an implicit particle current $J$ in the Ampère equation (19a) with an implicit electric field in the definition of $J$. Accordingly, an implicit electric field must be used in the Lorentz force defining the source of the momentum equation Eq. (19b). These requirements are met for $a=1$. Note that the scaling assumptions imply that the dimensionless Debye length also represents the scaled plasma period. Therefore, the uniform stability property with respect to $\lambda$ brings the stability of the method for time steps lager than the plasma period.

c) The density occurring in the Lorentz force is made explicit when the mass flux is implicit in order to uncouple the resolution of the Eqs. (19a) and (19b).

d) The consistency with the Gauss equation at the discrete level, requires the same level of implicitness for the mass flux in Eq. (19a) and the current $J$ in the Ampère equation (19d). This point will be detailed further in the sequel.

The linear stability proposed in [56] demonstrates that the AP property cannot be achieved with an implicitness level weaker than $(a, b, c)=(1,1,1)$. This choice defines a consistent discretization of the reformulated system (18). Indeed, Eqs. (19c), (19d) and (19b) in which the correction is omitted yield

$$
\begin{aligned}
\frac{\lambda^{2}}{\Delta t^{2}}\left(\tilde{E}^{m+1}-E^{m}\right) & =\frac{1}{\Delta t}\left(\nabla \times B^{m}-J^{m}\right) \\
& -\nabla \times \nabla \times \tilde{E}^{m+1}-n^{m} \tilde{E}^{m+1}-\nabla \cdot S^{m}+J^{m} \times B^{m} .
\end{aligned}
$$

Owing that the Ampère equation is initially verified: $\nabla \times B^{m}-J^{m}=\frac{\lambda^{2}}{\Delta t}\left(E^{m}-E^{m-1}\right)$, the following identity holds

$$
\begin{aligned}
\frac{\lambda^{2}}{\Delta t^{2}}\left(\tilde{E}^{m+1}-2 E^{m}+E^{m-1}\right)+\nabla \times & \times \times \tilde{E}^{m+1}+ \\
& n^{m} \tilde{E}^{m+1}+\nabla \cdot S^{m}-J^{m} \times B^{m}=0,
\end{aligned}
$$

which defines a time semi discretization of the reformulated Ampère equation (18b). A similar result can be obtained for the Gauss equation, with Eqs. (19a), (19b), (19e), (19f) and (20) providing

$$
\begin{aligned}
&-\nabla \cdot\left(\left(\frac{\lambda^{2}}{\Delta t^{2}}+n^{m}\right) \nabla p\right)=\frac{1}{\Delta t^{2}}\left(1-\tilde{n}^{m+1}-\lambda^{2} \nabla \cdot E^{m}\right)+\frac{1}{\Delta t} \nabla \cdot J^{m} \\
&+\nabla^{2}: S^{m}-\nabla \cdot\left(J^{m} \times B^{m}\right)+\nabla \cdot\left(n^{m} \tilde{E}^{m+1}\right),
\end{aligned}
$$


where $\tilde{n}^{m+1}=n^{m+1}+\Delta t^{2} \nabla \cdot\left(n^{m} \nabla p\right)$. Assuming that the Gauss and the continuity equations are satisfied at the previous time step $\lambda^{2} \nabla \cdot E^{m}=1-n^{m}$ and $\Delta t \nabla \cdot J^{m}=n^{m}-n^{m-1}$ the following identity holds

$$
\begin{aligned}
&-\nabla \cdot\left(\left(\frac{\lambda^{2}}{\Delta t^{2}}+n^{m}\right) \nabla p\right)=\frac{1}{\Delta t^{2}}\left(-\tilde{n}^{m+1}+2 n^{m}-n^{m-1}\right) \\
&+\nabla^{2}: S^{m}-\nabla \cdot\left(J^{m} \times B^{m}\right)+\nabla \cdot\left(n^{m} \tilde{E}^{m+1}\right) .
\end{aligned}
$$

This defines a time discretization of the reformulated Gauss equation (18b) provided that the correction at time level $m$ and $m-1$ vanishes.

Remark 2.2. In the semi discrete system (19), the Ampère and Gauss equations are not formulated with second order time derivatives as their continuous counterparts. They are solved thanks to Eqs. (20) and (21). However, implicit time discretizations are used in order to recover the consistency with the continuous reformulated system (18) as demonstrated by Eqs. (20-22). Another approach would consist in deriving the discrete system as a discretization of the reformulated continuous system. These two different methodologies have been implemented in the frame of the Vlasov-Poisson system giving rise to the PIC$A P 1$ and PIC-AP2 schemes proposed in [52, 53]. Working the discrete equations (20) and (21) gains the advantage to remove the need of a second initial condition, which may be difficult to derive. This is thus the path followed in this document and most of the related works.

\subsubsection{Overview of the spatial discretizations}

Different space discretizations have been considered. For kinetic description, either Particle-In-Cell [52, 53, 49] or semi-Lagrangian [7] discretizations have been implemented, while, for fluid descriptions finite volume (on Cartesian meshes) is used $[41,56]$. In this last series of works dedicated to the Euler-Maxwell system, an exact consistency with the Gauss equation can be obtained. To this end, the numerical flux associated to the mass flux must be used to construct the current of particles used in the Ampère equation. This property is sketched in the next lines in a simplified one-dimensional framework, with $B_{x}=0$. We refer to [56] for the details. Denoting $\mathcal{F}_{k+1 / 2}^{m+1}$ the numerical flux associated to the mass flux evaluated at the center of the cell $k, n_{k}^{m}$ and $\left.E_{x}^{m}\right|_{k+1 / 2}$ being the density and the electric field at time $t^{m}=m \Delta t$, with $\Delta t$ and $\Delta x$ the time and space mesh intervals, a discretization of the system (19a) and $(19 \mathrm{~d})$ is written as

$$
\begin{array}{r}
n_{k}^{m+1}=n_{k}^{m}+\frac{\Delta t}{\Delta x}\left(\mathcal{F}_{k+1 / 2}^{m+1}-\mathcal{F}_{k-1 / 2}^{m+1}\right), \\
\lambda^{2} \frac{1}{\Delta t}\left(\left.E_{x}^{m+1}\right|_{k+1 / 2}-\left.E_{x}^{m}\right|_{k+1 / 2}\right)=\mathcal{F}_{k+1 / 2}^{m+1} .
\end{array}
$$


Evaluating Eq. (24) at the cell interfaces $x_{k+1 / 2}$ and $x_{k-1 / 2}$ together with (23) yields

$$
\lambda^{2} \frac{1}{\Delta x}\left(\left.E_{x}\right|_{k+1 / 2} ^{m+1}-\left.E_{x}\right|_{k-1 / 2} ^{m+1}\right)+\left.n\right|_{k} ^{m+1}=\lambda^{2} \frac{1}{\Delta x}\left(\left.E_{x}\right|_{k+1 / 2} ^{m}-\left.E_{x}\right|_{k-1 / 2} ^{m}\right)+\left.n\right|_{k} ^{m} .
$$

This expression defines a discretization of the equation $\frac{\partial}{\partial t}\left(\lambda^{2} \partial E_{x} / \partial x\right)=-\frac{\partial n}{\partial t}$.

A similar property cannot be obtained with standard PIC methods. Indeed the implicit computation of the electric field is achieved by predicting the particles motion thanks to implicit discretization of the moments of the kinetic equations. The macroscopic quantities predicted on the grid are not exactly realized by the particles, which creates deviations between these quantities, preventing an exact consistency with the Gauss equation, although the implementation of the correction. This point is analyzed in [49].

\subsection{Related works}

Pioneering works on Asymptotic-Preserving methods for the quasi-neutral limit have been first devoted to the Euler-Poisson system [41], then extended to kinetic electrostatic descriptions by means of the Vlasov-Poisson system [52, $53,7]$. Electromagnetic fields have been considered in the frame of the bifluid isothermal Euler-Maxwell system in [56] (extended to the M1-Maxwell model in [80]) and finally with the Vlasov-Maxwell system [49] (see figure 2).

Some analogies exist with implicit methods, specifically in the framework of Particle-In-Cell discretizations for kinetic plasma descriptions, including the direct implicit methods $[105,35,34,89]$ and the moment implicit methods [116, $20,145,117,128]$. These methods have been derived to perform numerical simulations on large scales without the constraints of explicit methods, with time steps limited by the plasma period $\omega_{p, e}^{-1}$ and the mesh sizes by the Debye length. These are commonly the most stringent restrictions on the discretization parameters that are also removed thanks to AP-methods. Indeed, the scaling relations defining the quasi-neutral regime imply that, beside the dimensionless Debye length and the ratio of the reference velocity to the speed of light, the asymptotic parameter $\lambda$ carries the scaled plasma period $\bar{t} \omega_{p, e}^{-1}$ as well. Indeed, the following identity $\lambda^{2}=\lambda_{D}^{2} / \bar{x}^{2}=M^{2} /\left(\bar{t} \omega_{p, e}^{-1}\right)^{2}$, together with the assumption $M=1$ proves the above assertion. The uniform stability with respect to $\lambda$ ensures that AP-methods remain stable for discretization that do not resolve the plasma period, hence common properties with implicit or semi implicit methods outlined in more details in [49, section 4]. We also refer to [115] for related works on asymptotic limits of the Maxwell system.

\section{Drift limit for fluid descriptions of hot plasmas under large mag- netic fields}

\subsection{Introduction}

This section is devoted to the design of fluid models and numerical methods for thermal plasmas evolving under a strong magnetic field. The targeted appli- 


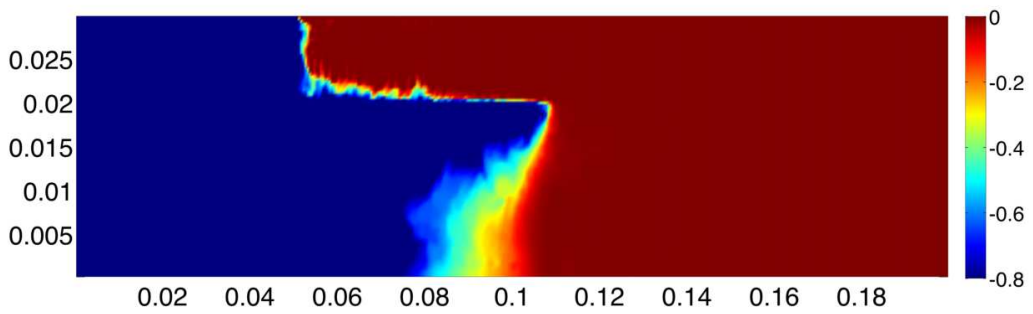

(a) Magnetic field at time $t=3.67 \mathrm{~ns}$.

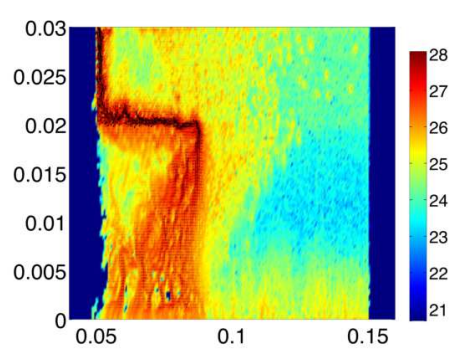

(b) Current density at $t=2.58 \mathrm{~ns}$.

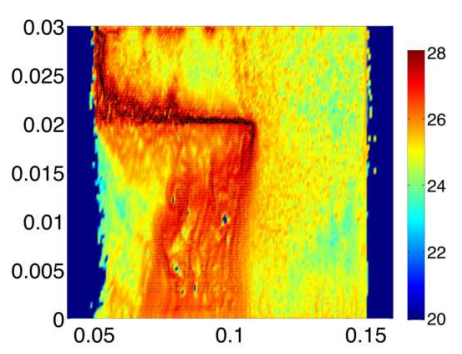

(c) Current density at $t=3.67$ ns.

Figure 2: Propagation of a magnetic choc wave into a dense plasma simulated thanks to the Vlasov-Maxwell system discretized by an Asymptotic-Preserving Particle-In-Cell method in the quasi-neutral limit. These computations are carried out with $\lambda_{D} / h \sim 2 \cdot 10^{-4}, h$ being the typical space step; (a) Magnetic field magnitude (Tesla) and (b), (c) electronic current density $\left(\mathrm{A} \cdot \mathrm{m}^{-2}\right.$, in decimal log-scale) as functions of the space coordinates (see [49]).

cations are tokamak plasmas and magnetically confined fusion [31, 119, 136, 75]. Its principle consists in heating a plasma to hundreds of thousands of degrees in order for the thermal agitation to overcome the Coulomb repulsion. Indeed, for the nuclear reaction to occur, the distance between the nuclei has to be lower than $10^{-15} \mathrm{~m}$. Simultaneously, the plasma expansion is prevented by confining the particles thanks to an intense magnetic field.

This section presents asymptotic preserving methods developed for simulating these hot plasmas evolving in the presence of strong magnetic fields. The magnetization of the plasma induces a severe anisotropy, with different parallel and perpendicular (with respect to the magnetic field) dynamics. The dynamic of interest is the perpendicular one, which is, compared to the parallel dynamic, a low frequency regime driven by the drift waves. Along the magnetic field lines, the acoustic waves are very fast, balancing almost instantly the electric field in order to enforce a zero force regime. The first purpose of the AP methods described in this section is to follow the slow perpendicular dynamic while accounting for the parallel force balance as well. The second aim is to explore the effectiveness of numerical methods that do not use coordinate systems adapted to the magnetic field geometry by contrast to standard approaches (see for instance $[6,69,71,83,123,127,131,132,4,86])$. Indeed standard approaches have some difficulties in specific areas such as the so-called "O point" or "X 
point" $[84,72,133]$ where the coordinate system is singular. Moreover, the goal pursued here is to easily account for changes in the magnetic field topology, with for instance the creation of magnetic islands [16, 8, 86, 90, 65, 146, 129, 147]. To this end, the numerical methods developed within this section are free from any assumption relating the magnetic field to the mesh or the coordinate system.

The collisions within tokamaks are weak, therefore the reliability of fluid descriptions is questioned [79] especially in the case of devices such as ITER and DEMO, with investigations [70] demonstrating that fluid models underestimate instability thresholds and overestimate turbulent fluxes. However, numerous fluid descriptions are implemented for tokamak plasmas [113, 94], in particular for the study of the plasma edge physics (see for instance $[71,139,25]$ ). Additionally, kinetic corrections may be formulated for fluid models in order to correct some of their flaws [79]. The main argument, however, seems to be the huge cost needed for the numerical resolution of kinetic models which is challenging in term of computational time as well as memory usage. Even in the gyro-kinetic approximation [110, 77, 25] kinetic models consider a distribution function in a five dimensional phase-space. Though their accuracy may be improved, fluid models provide access to a rich physics which helps understanding the various regimes that prevail within tokamaks and allows for the study of instability mechanisms [118, 136, 15].

The purpose of this section is thus to detail the Asymptotic-Preserving methodology in this framework. The plasma is described by two sets of fluid equations. The density, momentum and energy associated to the electrons and the ions are denoted $\left(n_{\alpha}, q_{\alpha}, W_{\alpha}\right)$ ( $\alpha=e$ for the electrons and $i$ for the ions), the charge of the particle being denoted $\mathfrak{q}_{\alpha}$. These quantities verify the EulerLorentz system

$$
\begin{aligned}
& \frac{\partial n_{\alpha}}{\partial t}+\nabla \cdot q_{\alpha}=0 \\
& m_{\alpha}\left(\frac{\partial q_{\alpha}}{\partial t}+\nabla \cdot\left(q_{\alpha} \otimes \frac{q_{\alpha}}{n_{\alpha}}\right)\right)+\nabla p_{\alpha}=\mathfrak{q}_{\alpha}\left(n_{\alpha} E+q_{\alpha} \times B\right), \\
& \frac{\partial W_{\alpha}}{\partial t}+\nabla \cdot\left(\left(W_{\alpha}+p_{\alpha}\right) \frac{q_{\alpha}}{n_{\alpha}}\right)=\mathfrak{q}_{\alpha} E \cdot q_{\alpha},
\end{aligned}
$$

where $k_{B}$ is Boltzmann constant, $m_{\alpha}$ the particle mass, $T_{\alpha}$ and $p_{\alpha}$ the temperature and the pressure of the gas, with the following expression of the energy

$$
W_{\alpha}=\frac{1}{2} m_{\alpha} \frac{\left|q_{\alpha}\right|^{2}}{n_{\alpha}}+\frac{3}{2} p_{\alpha}, \quad p_{\alpha}=n_{\alpha} k_{B} T_{\alpha} .
$$

The given magnetic field is assumed static, i.e. satisfying

$$
\frac{\partial B}{\partial t}=0, \quad \nabla \cdot B=0
$$

The electronic and ionic conservation equations are coupled to the electro-static field defined as

$$
-\Delta \phi=\frac{\mathfrak{q}}{\varepsilon_{0}}\left(n_{i}-n_{e}\right), \quad \text { with } E=-\nabla \phi,
$$


with $\varepsilon_{0}$ the vacuum permeability and $\mathfrak{q}$ the elementary charge. The purpose here is to investigate the drift limit of this system also referred to as gyro-fluid approximation [57]. While the drift limit may be derived as a mass-less regime with a vanishing particle inertia, it is derived as a low Mach limit combined with an assumption of large magnetic field strengths. Therefore the drift limit shares some analogies with the low-Mach regime and the difficulty investigated here consists in the transition of this low Mach flow which prevails in the plasma core, to regimes with an increased mean velocity and a lower temperature. This dynamical transition is observed in the Scrape-Off Layer (SOL), precisely in the magnetic pre-sheath and the sheath where the plasma temperature drops and the ions are accelerated by the electrostatic field [126, 137], the flow being potentially supersonic [33]. This section is organized as follows. The drift regime for the Euler-Lorentz system is stated, with the definition of the scaling relations. The magnetic field is assumed to be a given data that does not depend on time. Two Asymptotic-Preserving reformulations are thus proposed with, finally, the detailed computation of the self-consistent electric field.

\subsection{The Euler-Lorentz model in the drift regime}

In order to identify the drift regime, the system is rewritten using dimensionless variables. Denoting $\bar{n}, \bar{q}, \bar{T}$ the typical values for the plasma density,momentum, and temperature, the identities $n=\bar{n} n^{\prime}, q=\bar{q} q^{\prime}$ and $T=\bar{T} T^{\prime}$ hold for the physical $(n, q, T)$ and dimensionless $\left(n^{\prime}, q^{\prime}, T^{\prime}\right)$ quantities. The space and time scales of the observed phenomenon are $\bar{x}$ and $\bar{t}$, defining $\bar{u}=\bar{x} / \bar{t}$ the reference velocity. The re-scaled system is stated thanks to the following dimensionless parameters

$$
\left\{\begin{array}{l}
\varepsilon=\frac{m_{e}}{m_{i}}, \text { the electron to ion mass ratio } \\
\bar{t} \omega_{c}=\bar{t} \frac{\mathfrak{q} B}{m_{i}}, \text { the dimensionless ion cyclotron frequency } \\
\lambda=\frac{\lambda_{D}}{\bar{x}}, \text { the dimensionless Debye length; } \\
M^{2}=\frac{\bar{u}^{2}}{c_{s}^{2}}=\frac{\bar{u}^{2} m_{i}}{k_{B} \bar{T}}, \text { the squared ionic Mach number }
\end{array}\right.
$$

The number of free parameters is reduced thanks to scaling relations defining the regime in which the system is observed. In this aim, the scales of interest are precised in Tab. 1.

The following scaling defines the drift regime investigated in the sequel:

$$
\bar{u} \sim \frac{\bar{q}}{\bar{n}}, \quad \bar{E} \sim \bar{u} \bar{B}, \quad \frac{1}{M^{2}} \sim \bar{t} \omega_{c} \sim \frac{1}{\tau} .
$$

The reference velocity is the mean plasma velocity. The plasma is close to quasi-neutrality $(\lambda \ll 1)$, hence the frozen field scaling discussed in Sec. 2.1 (see Eq. (7)). Finally, the Mach number and the reciprocal of the dimensionless 
Table 1: Characteristics of the ITER and DEMO tokamaks $[114,75]$ and typical values of the dimensionless parameters.

\begin{tabular}{|l|c|c|}
\hline & ITER & DEMO \\
\hline \hline External radius $(\mathrm{m})$ & 6.2 & $6.1-9.55$ \\
\hline Inner radius $(\mathrm{m})$ & \multicolumn{2}{|c|}{2} \\
\hline \hline Magnetic field intensity $(\mathrm{T})$ & 5.3 & 7 \\
\hline \hline Plasma density $\left(\mathrm{m}^{-3}\right)$ & $10^{20}$ & $1.510^{20}$ \\
\hline Plasma Temperature $(\mathrm{eV})$ & \multicolumn{2}{|c|}{$10^{4}$} \\
\hline \hline Discharge duration $(\mathrm{s})$ & 400 & 1000 \\
\hline
\end{tabular}

\begin{tabular}{|c|c|}
\hline \multicolumn{2}{|c|}{ Dimensionless Par. } \\
\hline \hline$\varepsilon$ & $10^{-4}$ \\
\hline \hline$\lambda$ & $10^{-5}$ \\
\hline \hline$\left(\bar{t} \omega_{c}\right)^{-1}$ & $10^{-6}$ \\
\hline \hline$M^{2}$ & $10^{-8}$ \\
\hline
\end{tabular}

ion cyclotron frequency define two small scales defining the force balance in the limit regime. To simplify the presentation these two small scales are unified in a single parameter denoted $\tau$.

The Euler-Lorentz-Poisson system (25-26) can thus be written using three dimensionless parameters $\varepsilon, \lambda$ and $\tau$. To simplify the notations, the subscript will be dropped for the ions, the electronic quantities being indexed by $e$. The re-scaled system writes

$$
\begin{aligned}
& \frac{\partial n}{\partial t}+\nabla \cdot q=0, \\
& \frac{\partial q}{\partial t}+\nabla \cdot\left(q \otimes \frac{q}{n}\right)+\frac{1}{\tau} \nabla p=\frac{1}{\tau}(n E+q \times B), \\
& \left.\frac{\partial W}{\partial t}+\nabla \cdot\left((W+p) \frac{q}{n}\right)\right)=E \cdot q, \\
& W=\tau \frac{1}{2} \frac{q^{2}}{n}+\frac{3 p}{2}, \quad p=n T .
\end{aligned}
$$

A similar system is written for the electrons

$$
\begin{gathered}
\frac{\partial n_{e}}{\partial t}+\nabla \cdot q_{e}=0, \\
\varepsilon\left(\frac{\partial q_{e}}{\partial t}+\nabla \cdot\left(q_{e} \otimes \frac{q_{e}}{n_{e}}\right)\right)+\frac{1}{\tau} \nabla p_{e}=-\frac{1}{\tau}\left(n_{e} E+q_{e} \times B\right), \\
\left.\frac{\partial W_{e}}{\partial t}+\nabla \cdot\left(\left(W_{e}+p_{e}\right) \frac{q_{e}}{n_{e}}\right)\right)=-E \cdot q_{e}, \\
W_{e}=\varepsilon \tau \frac{1}{2} \frac{q_{e}^{2}}{n_{e}}+\frac{3 p_{e}}{2}, \quad p_{e}=n_{e} T_{e} .
\end{gathered}
$$

The electric field is provided by

$$
-\lambda^{2} \Delta \phi=n-n_{e}, \quad E=-\nabla \phi .
$$

The quasi-neutrality is recovered from the Poisson equation (31) in the limit $\lambda \rightarrow 0$. The Poisson equation cannot be used to compute the electric potential 
in this regime. Two alternatives have been investigated and detailed in Sec. 3.5. The drift limit consists in letting $\tau \rightarrow 0$. This limit is singular, the momentum equation (29b) being degenerate for $\tau=0$. To highlight this feature, some notations are introduced.

Notations. $B$ denoting the magnetic field, $b$ the unit vector pointing in the direction of $B$, with $b:=B /|B|$, for all scalar $p$ and vector $q$, we define

$$
\begin{array}{ll}
q_{\|}:=(q \cdot b), & q_{\perp}:=q-b q_{\|}=(\mathbb{I} d-b \otimes b) q=b \times q \times b, \\
\nabla_{\|} p:=b \cdot \nabla p, & \nabla_{\perp} p:=(\mathbb{I} d-b \otimes b) \nabla p=\nabla p-(\nabla p \cdot b) b, \\
\nabla_{\|} \cdot q_{\|}:=\nabla \cdot\left(q_{\|} b\right), & \nabla_{\perp} \cdot q_{\perp}:=\nabla \cdot\left(q_{\perp}\right) .
\end{array}
$$

Inserting formally $\tau=0$ into (29) yields to

$$
\begin{aligned}
& \frac{\partial n}{\partial t}+\nabla \cdot q=0 \\
& \nabla p=n E+q \times B, \\
& \left.\frac{\partial W}{\partial t}+\nabla \cdot\left((W+p) \frac{q}{n}\right)\right)=E \cdot q, \quad W=3 / 2 p .
\end{aligned}
$$

Eq. (33b) translates a "zero force regime", that does not allow for the computation of the parallel momentum. Indeed, projecting onto the parallel and perpendicular directions, the following balances occur

$$
\begin{aligned}
& q_{\perp}=\frac{b}{|B|} \times(-n E+\nabla p), \\
& \nabla_{\|} p=n E_{\|},
\end{aligned}
$$

In the drift regime, the perpendicular momentum component $q_{\perp}$ instantly adjusts to cancel the perpendicular electric and pressure forces. These two forces give rise to the classical drift velocities, namely the " $E \times B$ " $\operatorname{drift}(E \times B) /|B|^{2}$ and the diamagnetic drift $-(\nabla p \times B) /\left(n|B|^{2}\right)$.

Along the magnetic field lines, the electric field balances the pressure gradient and the system becomes singular for the computation of the parallel momentum. To understand more precisely the parallel dynamic, it is informative to establish the acoustic wave equation. Using Eq. (29c) together with (29b), provides an equation for the ionic pressure

$\frac{3}{2} \frac{\partial^{2} p}{\partial t^{2}}+\nabla \cdot\left(H \frac{\partial q_{\|}}{\partial t}\right)=-\nabla_{\perp} \cdot\left(H \frac{\partial q_{\perp}}{\partial t}\right)-\nabla \cdot\left(\frac{\partial H}{\partial t} q\right)+\frac{\partial}{\partial t}(E \cdot q)-\frac{\partial^{2}}{\partial t^{2}}\left(\frac{\tau(q)^{2}}{2 n}\right)$,

where $H=W+p / n$ is the system enthalpy. Projecting Eq. (29b) onto the magnetic field lines gives

$$
\frac{\partial}{\partial t} q_{\|}+\nabla \cdot\left(q \otimes \frac{q}{n}\right)_{\|}=\frac{1}{\tau}\left(-\nabla_{\|} p+n E_{\|}\right),
$$


which leads to

$$
\begin{aligned}
\frac{3}{2} \frac{\partial^{2} p}{\partial t^{2}}-\frac{1}{\tau} \nabla & \cdot(H(b \otimes b)(\nabla p-n E))=-\nabla \cdot\left((b \otimes b)\left(q \otimes \frac{q}{n}\right)\right) \\
& -\nabla \cdot\left(H \frac{\partial q_{\perp}}{\partial t}\right)-\nabla \cdot\left(\frac{\partial H}{\partial t} q\right)+\frac{\partial}{\partial t}(E \cdot q)-\frac{\partial^{2}}{\partial t^{2}}\left(\frac{\tau(q)^{2}}{2 n}\right) .
\end{aligned}
$$

This equation reveals a speed of sound along the magnetic field lines scaling as $1 / \sqrt{\tau}$, which demonstrate that in the limit $\tau \rightarrow 0$ the pressure waves travel at infinite speed to adjust instantly to the electric force, securing thus the force balance.

Two difficulties need to be overcome in order to efficiently address the drift regime in the context of tokamaks.

The first one relates to the vanishing of the inertia in the momentum equation which prevents from computing the parallel momentum explicitly. Two alternatives have been proposed. The first one consists in using the aligned momentum as the Lagrange multiplier of the parallel force balance. The second one is constructed on ideas borrowed from the low Mach regime [63, 82] with a computation of the pressure securing the force balance along the magnetic field lines. By these means, the singularity of the problem can be overcome at the price of the resolution of an anisotropic diffusion problem.

The second difficulty addressed here is related to the periodicity of the torus containing the plasma. The anisotropic diffusion equations derived to transform the drift limit into a regular limit are supplemented with periodic boundary conditions at each end of the magnetic field lines. This defines an ill posed problem in the limit regime. This is a characteristic issue for tokamak simulations, with highly magnetized plasmas evolving in a periodic geometry. For this class of anisotropic problems, Asymptotic-Preserving techniques have been developed. Their presentation is postponed to Section 4 .

\subsection{Momentum based reformulation}

This approach makes use of the parallel momentum as a Lagrange multiplier associated to the parallel force balance (34b). The method is sketched for the ions, the equation (29b) providing

$$
\frac{\partial^{2}}{\partial t^{2}} q_{\|}-\frac{1}{\tau}\left(-\frac{\partial \nabla_{\|} p}{\partial t}+\frac{\partial\left(n E_{\|}\right)}{\partial t}\right)=-\frac{\partial}{\partial t}\left(\nabla \cdot \mathbb{S}_{\|}\right), \quad \mathbb{S}=q \otimes \frac{q}{n} .
$$

Working the energy equation (29c), the following identity can be written

$$
\begin{aligned}
\frac{\partial \nabla_{\|} p}{\partial t}=-\nabla_{\|} \nabla \cdot & \left(\frac{2}{3} H q_{\|}\right)+\nabla_{\|} \mathcal{G}, \\
\mathcal{G} & :=\frac{2}{3}\left(-\nabla \cdot\left(H q_{\perp}\right)+E \cdot q-\frac{\partial}{\partial t}\left(\frac{\tau(q)^{2}}{2 n}\right)\right) .
\end{aligned}
$$

Substituting the left hand side of this equation into Eq. (36) finally gives a wave like equation for the parallel momentum:

$$
\tau \frac{\partial^{2}}{\partial t^{2}} q_{\|}-\left(\nabla_{\|} \nabla \cdot\left(\frac{2 H}{3} q_{\|}\right)\right)=-\nabla_{\|} \mathcal{G}+\frac{\partial\left(n E_{\|}\right)}{\partial t}-\tau \frac{\partial}{\partial t}\left(\nabla \cdot \mathbb{S}_{\|}\right)
$$


Note that, in the drift limit, Eq. (38) guarantees the zero force regime along the magnetic field lines. Indeed, inserting $\tau=0$ into (38), we get

$$
-\left(\nabla_{\|} \nabla \cdot\left(\frac{2 H}{3} q_{\|}\right)\right)=-\nabla_{\|} \mathcal{G}+\frac{\partial\left(n E_{\|}\right)}{\partial t},
$$

which, owing to (37) reduces to

$$
\frac{\partial}{\partial t}\left(\nabla_{\|} p-n E_{\|}\right)=0
$$

Remark 3.1. The second order operator involved in Eq. (38) is non standard. It is constructed as the gradient of a divergence. However, being applied to a scalar field, it translates the double derivative in the parallel direction. This equation is therefore well posed provided it is supplemented with adequate boundary conditions.

\subsection{Pressure based reformulation}

Another approach proposed in [48] and implemented in [23] solves the acoustic equation in order to impose that the force imbalance along the magnetic field lines is bounded by $\tau$, with

$$
-\nabla_{\|} p+n E_{\|}=\mathcal{O}(\tau) .
$$

This equilibrium is computed in order to prevent the degeneracy of the parallel momentum equation, with, in the drift limit, the set of equations

$$
\begin{aligned}
& \frac{\partial n}{\partial t}+\nabla \cdot q=0, \\
& q_{\perp}=\frac{b}{|B|} \times(n E+\nabla p), \\
& \frac{\partial}{\partial t} q_{\|}+\nabla \cdot\left(q \otimes \frac{q}{n}\right)_{\|}=\lim _{\tau \rightarrow 0}\left(\frac{1}{\tau}\left(-T \nabla_{\|} n+n E_{\|}\right)\right),
\end{aligned}
$$

providing all the quantities, including $q_{\|}$if the condition (40) is met.

This procedure has only been developed in the context of an isothermal plasma description. The set of equations considered does not incorporate the energy equation and the pressure gradient reduces to $\nabla p=T \nabla n$. The reformulation is derived from the momentum conservation (29b) projected along the magnetic field lines

$\nabla_{\|} \cdot \frac{\partial q_{\|}}{\partial t}=\frac{1}{\tau} \nabla_{\|} \cdot\left(-T \nabla_{\|} n+n E_{\|}\right)-\nabla_{\|}^{2}: \mathbb{S}, \quad \nabla_{\|}^{2}: \mathbb{S}:=\nabla_{\|} \cdot\left(\nabla \cdot\left(q \otimes \frac{q}{n}\right)_{\|}\right)$.

together with the density conservation (29a), rather than the energy equation (29c) (see section 3.2) yielding to the equation of the acoustic waves for isothermal descriptions

$$
\tau \frac{\partial^{2} n}{\partial t^{2}}-\nabla_{\|} \cdot\left(T \nabla_{\|} n\right)=\nabla_{\|} \cdot\left(n E_{\|}\right)-\tau\left(\nabla \cdot q_{\perp}-\nabla_{\|}^{2}: \mathbb{S}\right) .
$$


This last equation provides a way to compute the plasma density which secures Condition (40). This reformulation is implemented and validated in [23]. It borrows some of the concepts of low-Mach regime numerical methods (see for instance [63]) that could be transposed to this framework.

\subsection{Self-consistent electric field computation}

The difficulty stemming from the computation of the electric field is related to the quasi-neutrality assumption. This is the issue addressed in Sec. 2.3.2 with the degeneracy of the Poisson equation (31) in the limit $\lambda \rightarrow 0$. This equation degenerates into $\rho=0$ and therefore cannot provides a means of computing the electric potential.

To derive the equation verified by the electric potential $\phi$, the conservation of the ionic (29a) and electronic (30a) densities are combined to provide the continuity equation defined as

$$
\frac{\partial \rho}{\partial t}+\nabla \cdot J=0
$$

with $\rho=n-n_{e}$ and $J=q-q_{e}$. In the quasi-neutral limit, this equation degenerate into the constraint $\nabla \cdot J=0$. Time differentiating this identity, yields

$$
\nabla \cdot\left(\frac{\partial q}{\partial t}-\frac{\partial q_{e}}{\partial t}\right)=0
$$

so that the momentum equations can be used to introduce a contribution of the electric field into this relation by means of the electric force definition. This is similar to the reformulation derived in the section 2.3.2 and detailed in the next lines thanks to a time semi-discretization of the system following [23]

$$
\begin{aligned}
& \frac{1}{\Delta t}\left(n^{m+1}-n^{m}\right)+\nabla_{\|} \cdot q_{\|}^{m+1}+\nabla \cdot q_{\perp}^{m}=0, \\
& \frac{1}{\Delta t}\left(q^{m+1}-q^{m}\right)+\nabla \cdot \mathbb{S}+\frac{1}{\tau} T \nabla n^{m+1}= \\
& \frac{1}{\tau}\left(n^{m+1} \nabla \phi^{m+1}+q^{m+1} \times B\right), \\
& \frac{1}{\Delta t}\left(q_{e}^{m+1}-q_{e}^{m}\right)+\nabla \cdot \mathbb{S}_{e}+\frac{1}{\varepsilon \tau} T \nabla n^{m+1}= \\
& -\frac{1}{\varepsilon \tau}\left(n^{m+1} \nabla \phi^{m+1}+q_{e}^{m+1} \times B\right) .
\end{aligned}
$$

On the discrete level, the quasi-neutrality constraint can be discretized as

$$
\nabla \cdot\left(q^{m+1}-q_{e}^{m+1}\right)=0,
$$

giving rise to an equation for $\phi^{m+1}$

$$
-\nabla_{\|} \cdot\left(n^{m+1}\left(1+\varepsilon^{-1}\right) \nabla_{\|} \phi^{m+1}\right)=\tau \mathcal{S} .
$$

We refer to [23] for the detailed algebra and the expression of $\mathcal{S}$. 
An alternative is proposed in [66]. It relies on the so-called Boltzmann relation for the electrons. This approximation consists in neglecting the particle inertia in the electronic momentum equation (30), which amounts to letting $\varepsilon \rightarrow 0$, assuming a temperature with vanishing gradients in the parallel direction. This last assumption is justified by the heat flux responsible for a rapid balancing of the temperature along the magnetic field lines [21, 93]. With these assumptions, the electronic momentum equation gives rise to the following equilibrium

$$
T_{e} \nabla_{\|} n_{e}=n_{e} \nabla_{\|} \phi
$$

Assuming the quasi-neutrality of the plasma $n=n_{e}$, the Maxwell-Boltzmann relation [106] can be stated

$$
n=n_{0} \exp \left(\frac{\phi}{T}\right),
$$

with $n_{0}$ an equilibrium density verifying $\nabla_{\|} n_{0}=0$. This system closure is used to derive an equation for the electric potential that is not degenerate in the quasi-neutral limit. Indeed, inserting Eq. 46 into Eq. (30) yields

$$
-\lambda^{2} \Delta \phi=n-n_{0} \exp \left(\frac{\phi}{T}\right) .
$$

A second occurrence of the electric field is introduced into the Poisson equation which prevents its degeneracy in the quasi-neutral limit. This property is thoroughly analyzed in [61].

\subsection{Related works}

The first implementation of the "parallel momentum" reformulation is proposed in [55] for an isothermal description of the plasma with an external constant electric field. The set up does not exactly suit tokamaks requirements, the periodicity of the domain is indeed not addressed in this first work. For isotherm descriptions, the energy equation being dropped out in the model, the reformulation is therefore derived thanks to the density and the momentum equations. This equation is supplemented with Dirichlet boundary conditions and discretized in a two dimensional computation domain with a magnetic field aligned with one coordinate.

The generalization to magnetic field geometries uncorrelated to either the coordinate system or the mesh in first addressed in [22]. The Dirichlet boundary conditions are substituted with Neumann ones, reproducing the difficulty to account for the periodicity of the torus. This brings a real difficulty in developing efficient numerical methods since the anisotropic equations stemming from the reformulation are not well posed in the drift limit $\tau \rightarrow 0$. This issue is overcome thanks to the "solution via the primitive" method described in section 4.3.

The "Pressure based" reformulation, proposed in [48] has been developed for isothermal descriptions with one species of particles (the ions being thus assumed at rest) in $[55,22]$. It has been brought to a more elaborated context in [66], for 
a complete set of equations for the ions, incorporating an energy equation, gyroviscous terms from the Braginskii closure [21] and an adiabatic response for the electron, the so-called Boltzmann relation (see section 3.5). The magnetic field is constant and aligned with one coordinate, periodic boundary conditions being prescribed at each end of the field lines. The anisotropic diffusion equation stemming from the reformulation is solved thanks to the duality based AP method (see section 4.2) for the three dimensional simulation of the slab ion temperature gradient instability (ITG [79, 101, 90, 87]) as depicted in figure 3

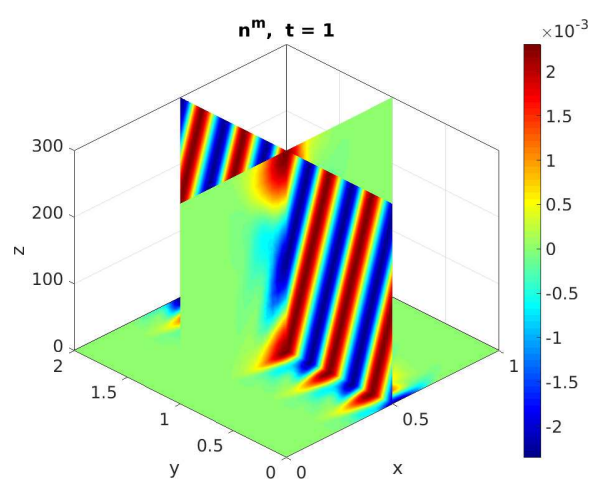

(a) Density at $\mathrm{t}=1$.

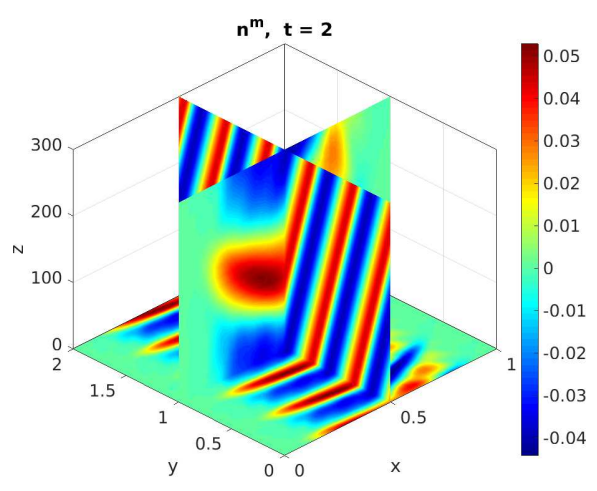

(b) Density at $t=2$.

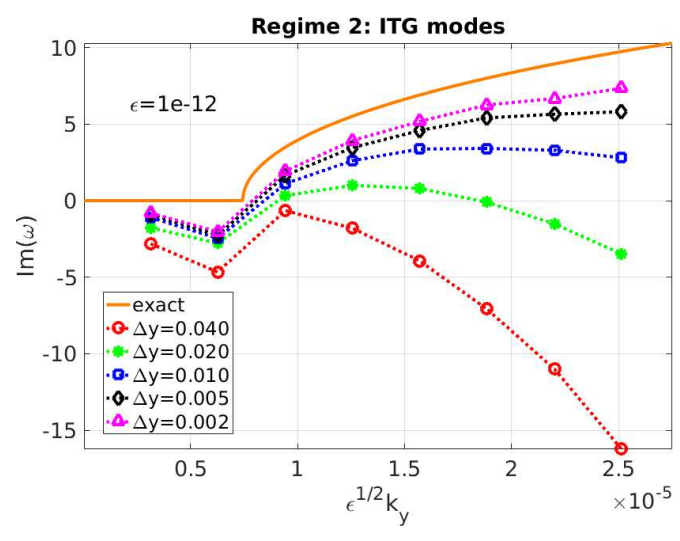

(c) ITG growth rate.
Figure 3: Three dimensional slab ITG simulation thanks to a numerical method implementing the parallel momentum reformulation on a $40 \times 200 \times 20$ mesh: density perturbation contour plots at times $\mathrm{t}=1$ (a) and $t=2$ (b) as functions of the space variables; and (c) ITG growth rate estimated from the simulations against the analytic values (solid line) as functions of the scaled wave number of the initial perturbation and for different mesh refinements (see the discussion in [66]).

\section{Numerical methods for strongly anisotropic elliptic and diffusion equations}

\subsection{Introduction}

This section is devoted to an overview of methods designed for the numerical resolution of elliptic (or diffusion) equations with large anisotropies. This is a class of problems representative of the difficulty stemming from the simulation 
of plasmas under a large magnetic field. Tokamak plasmas are a good example of this kind of problems. In this framework the difficulty lies in the periodic boundary conditions applied at the field line extremities in order to account for the periodicity of the torus. Other fields of application can be named, with for instance ionospheric plasma simulation [12] presenting the same difficulty, however with Neumann boundary conditions prescribed at each end of the magnetic lines.

The difficulty just mentioned is outlined on a simplified toy model, consisting of an anisotropic elliptic equation posed in a cuboid domain $\Omega_{x} \times \Omega_{z}$, the boundaries being $\Gamma_{x}=\partial \Omega_{x}$ and $\Gamma_{z}=\partial \Omega_{z}$. The anisotropy strength is denoted $\varepsilon$,

$$
\left(P^{\varepsilon}\right)\left\{\begin{array}{l}
-\frac{\partial}{\partial x}\left(A_{\perp} \frac{\partial \phi^{\varepsilon}}{\partial x}\right)-\frac{1}{\varepsilon} \frac{\partial}{\partial z}\left(A_{\|} \frac{\partial \phi^{\varepsilon}}{\partial z}\right)=f^{\varepsilon}, \quad \text { in } \Omega_{x} \times \Omega_{z}, \\
\frac{\partial \phi^{\varepsilon}}{\partial z}=0, \quad \text { on } \Gamma_{z} \times \Omega_{x}, \\
\phi^{\varepsilon}=0, \quad \text { on } \Gamma_{x} \times \Omega_{z},
\end{array}\right.
$$

$A_{\perp}$ and $A_{\|}$being two positive functions. In the Dynamo-3D model mentioned above, the electrostatic potential computed by means of the quasi-neutrality equation, verifies an analogous anisotropic elliptic equation, when the magnetic field is assumed aligned with the $z$ coordinate. The problem associated to the dominant operator in the limit of infinite anisotropy strength is ill-posed, its kernel being populated by the functions that do not depend on the $z$ coordinate. Indeed, multiplying (48) by $\varepsilon$ and considering formally the limit $\varepsilon \rightarrow 0$ yields

$$
(D)\left\{\begin{array}{l}
\frac{\partial}{\partial z}\left(A_{\|} \frac{\partial \phi^{0}}{\partial z}\right)=0, \quad \text { in } \Omega_{x} \times \Omega_{z}, \\
\frac{\partial \phi^{0}}{\partial z}=0, \quad \text { on } \Gamma_{z} \times \Omega_{x}, .
\end{array}\right.
$$

This degenerate system admits an infinite number of solutions namely all functions $\bar{\psi}$ only depending on $x$. However, $\phi^{0}$ defined as the limit of $\phi^{\varepsilon}$, the solution of the problem (48), verifies a well posed problem [54,50]. This system is obtained by integrating the elliptic equation (48) along the $z$ coordinate. Thanks to the boundary conditions applied on $\Gamma_{z}$, one can write, in the limit $\varepsilon \rightarrow 0$

$$
\left(P^{0}\right)\left\{\begin{array}{l}
-\frac{\partial}{\partial x}\left(\bar{A}_{\perp} \frac{\partial \phi^{0}}{\partial x}\right)=\bar{f}^{0}, \quad \text { in } \Omega_{x}, \\
\phi^{0}=0, \quad \text { on } \Gamma_{x} \times \Omega_{z} .
\end{array}\right.
$$

In this equation $\bar{f}$ is the mean value of $f$ along the $z$ direction:

$$
\bar{f}(x)=\frac{1}{\operatorname{mes}\left(\Omega_{z}\right)} \int_{\Omega_{z}} f(x, z) d z,
$$

and similarly for $\bar{A}_{\perp}$. 
The system (50) is obtained as the limit problem of the anisotropic equation. At this point the singular nature of the problem is clearly outlined. The limit problem (50) is a one dimensional elliptic problem integrated along the anisotropy direction, while the initial problem (48) is a two dimensional elliptic equation. For small values of the asymptotic parameter, standard discretizations of the singular perturbation problem (48) will become consistent with the degenerate problem (49). The conditioning of the system matrix is thus expected to blow up with vanishing $\varepsilon$-values as reported on the plots of Fig. 4(a). Jointly with the conditioning blow up, the precision of the numerical approxi-

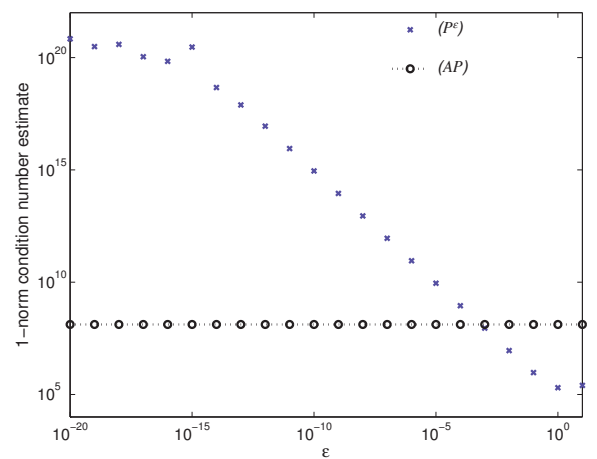

(a) Condition number estimate (mesh $50 \times$ $50)$.

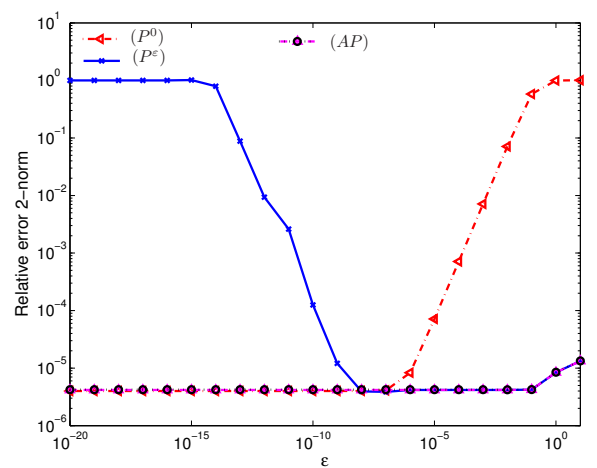

(c) Norm of the relative error between the exact solution and the numerical approximations $($ mesh $500 \times 500)$.

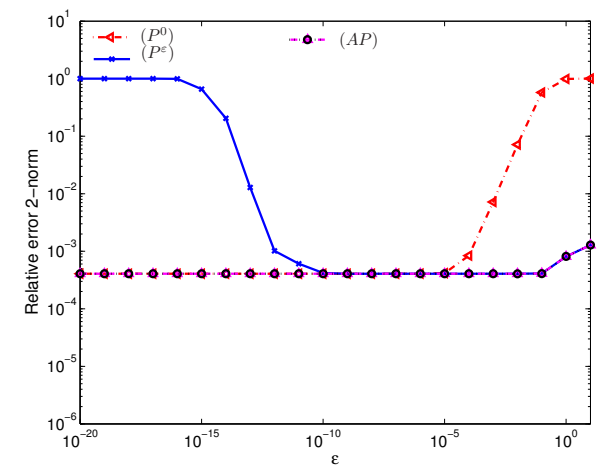

(b) Norm of the relative error between the exact solution and the numerical approximations $($ mesh $50 \times 50)$.

Figure 4: Property of standard discretizations of the singular perturbation problem (48) $\left(P^{\varepsilon}\right)$ and the limit problem $(50)\left(P^{0}\right)$ compared to an AP-method (AP) : condition number of the linear system obtained thanks to a $\mathbb{Q}_{1}$ finite element method, relative error computed between the exact solution and the numerical approximation as functions of the anisotropy strength $[54,11]$.

mation cannot be preserved for large anisotropy strength. This loss of accuracy is found for $\varepsilon$ values all the larger that the mesh is more refined, as shown by approximation errors displayed on Figs. 4(b) and 4(c).

The principle of AP-Schemes $[54,50,11,62,112,121]$ is to secure the consistency of the discrete system with the limit problem (50) when $\varepsilon \rightarrow 0$. In order to harness the microscopic information lost in the degenerate problem (49), these 
methods implement a decomposition of the solution into a part belonging to the kernel $\mathcal{G}$ of the dominant operator, supplemented by a correction, giving rise to

$$
\phi(x, z)=\bar{\phi}+\phi^{\prime}(x, z), \quad \forall(x, z) \in \Omega_{x} \times \Omega_{z},
$$

where $\bar{\phi} \in \mathcal{G}$ is the mean of the function $\phi$ as defined by (51) and $\phi^{\prime}$ is the fluctuating part verifying

$$
\bar{\phi}^{\prime}=0 \text {. }
$$

The difficulty lies now in the discretization of the properties verified by these two components. Different approaches have been developed and detailed in the next sections.

\subsection{Duality based reformulation}

The first implementation of the AP concepts have been carried out in [54] with a system consisting of an equation for the mean part of the solution coupled to an equation for the fluctuation:

$$
\begin{aligned}
& \left\{\begin{array}{c}
-\frac{\partial}{\partial x}\left(\bar{A}_{\perp} \frac{\partial \bar{\phi}}{\partial x}\right)=\bar{f}+\frac{\partial}{\partial x}\left(\overline{A_{\perp}^{\prime} \frac{\partial \phi^{\prime}}{\partial x}}\right), \quad \text { in } \Omega_{x}, \\
\bar{\phi}=0, \quad \text { on } \Gamma_{x},
\end{array}\right. \\
& \left\{\begin{array}{c}
-\varepsilon \frac{\partial}{\partial x}\left(A_{\perp} \frac{\partial \phi^{\prime}}{\partial x}\right)-\frac{\partial}{\partial z}\left(A_{\|} \frac{\partial \phi^{\prime}}{\partial z}\right)+\varepsilon \frac{\partial}{\partial x}\left(\overline{A_{\perp}^{\prime} \frac{\partial \phi^{\prime}}{\partial x}}\right)= \\
\varepsilon f^{\prime}+\varepsilon \frac{\partial}{\partial x}\left(A_{\perp}^{\prime} \frac{\partial \bar{\phi}}{\partial x}\right), \text { in } \Omega_{x} \times \Omega_{z}, \\
\partial_{z} \phi^{\prime}=0 \text { on } \Omega_{x} \times \Gamma_{z}, \quad \phi^{\prime}=0 \quad \text { on } \Gamma_{x} \times \Omega_{z}, \quad \overline{\phi^{\prime}}=0 \quad \text { in } \Omega_{x} .
\end{array}\right.
\end{aligned}
$$

In the limit $\varepsilon \rightarrow 0$ the degenerate problem (49) is recovered from the equation (53), however this equation is verified by only the fluctuating part rather than the entire solution. The zero mean property verified by the fluctuation restores the well posedness of the system for $\varepsilon=0$ with $\phi^{\prime}=0$ as unique solution. Inserting this identity into (52), the limit problem (50) is recovered. This demonstrates that, the limit $\varepsilon \rightarrow 0$ is regular in the reformulated system and consequently, that the formulation (52-53) is Asymptotic-Preserving.

When the anisotropy direction is aligned with one coordinate, the discretization of the functional space containing the mean function is straightforward. A weak formulation of the system (52) can be stated as

$$
\begin{aligned}
& \text { Find } \bar{\phi} \in \mathcal{G}:=\left\{\bar{\psi} \in H^{1}\left(\Omega_{x}\right) \mid \bar{\psi}=0 \text { on } \Gamma_{x}\right\} \\
& \left(A_{\perp} \partial_{x} \bar{\phi}, \partial_{x} \bar{\psi}\right)=(\bar{f}, \bar{\psi})+\left(\overline{A_{\perp}^{\prime} \partial_{x} \phi^{\prime}}, \partial_{x} \bar{\psi}\right), \quad \forall \bar{\psi} \in \mathcal{G} .
\end{aligned}
$$

with $(\phi, \psi):=\int_{\Omega} \phi \psi d x d z$. The task is more intricate for the discretization of the functional space $\mathcal{A}$ populated by the fluctuations. Introducing

$$
\mathcal{V}:=\left\{\psi^{\prime} \in H^{1}\left(\Omega_{x} \times \Omega_{z}\right) \mid \psi^{\prime}=0 \text { on } \Gamma_{x} \times \Omega_{z}\right\},
$$


a weak formulation of the problem (53) providing $\phi^{\prime}$ is

$$
\begin{gathered}
\text { Find } \phi^{\prime} \in \mathcal{A}:=\left\{\psi^{\prime} \in \mathcal{V} \mid \bar{\psi}^{\prime}=0\right\} \\
\left(A_{\|} \partial_{z} \phi^{\prime}, \partial_{z} \psi^{\prime}\right)+\varepsilon\left(A_{\perp} \partial_{x} \phi^{\prime}, \partial_{x} \psi^{\prime}\right)-\varepsilon\left(\overline{A_{\perp} \partial_{x} \phi^{\prime}}, \partial_{x} \psi^{\prime}\right)= \\
\varepsilon\left(f^{\prime}, \psi^{\prime}\right)-\varepsilon\left(A_{\perp}^{\prime} \partial_{x} \bar{\phi}, \partial_{x} \psi^{\prime}\right), \quad \forall \psi^{\prime} \in \mathcal{A} .
\end{gathered}
$$

The discretization of the functional space $\mathcal{A}$ is quite intricate. It is indeed not straightforward to construct a basis of finite element functions that guaranty the zero mean property of the fluctuations. An alternative approach is therefore implemented, thanks to the introduction of a Lagrangian aimed at penalizing the zero mean constraint. The weak formulation of the problem can thus be recast into

Find $\phi^{\prime} \in \mathcal{V}$ and $\bar{P} \in \mathcal{G}$ such that

$$
\left\{\begin{aligned}
\left(A_{\|} \partial_{z} \phi^{\prime}, \partial_{z} \psi\right)+\varepsilon\left(A_{\perp} \partial_{x} \phi^{\prime}, \partial_{x} \psi\right)-\varepsilon\left(\overline{A_{\perp} \partial_{x} \phi^{\prime}}, \partial_{x} \psi\right)+(\bar{P}, \psi) & = \\
\varepsilon\left(f^{\prime}, \psi\right)-\varepsilon\left(A_{\perp}^{\prime} \partial_{x} \bar{\phi}, \partial_{x} \psi\right), \quad \forall \psi \in \mathcal{V}, & \\
\left(\bar{\chi}, \phi^{\prime}\right)=0, \quad \forall \bar{\chi} \in \mathcal{G} &
\end{aligned}\right.
$$

In the weak formulation (55) the fluctuations are functions of the space $\mathcal{V}$ which is readily discretized thanks to classical methods. Note that if $\phi^{\prime}$ verify (53) then $\left(\phi^{\prime}, 0\right)$ is the solution of $(55)$. Conversely if $\left(\phi^{\prime}, \bar{P}\right)$ is the solution of $(55)$, then choosing a test function $\psi$ verifying $\bar{\psi}=0$, and noting that the right hand side of Eq. (55) has a zero mean, it is straightforward to prove that $\bar{\phi}^{\prime}=0$. We refer to [54] for more details and the demonstration of the equivalence of the reformulated system $(54),(55)$ with the singular perturbation problem (48).

A finite element discretization of the system (54),(55) gives rise to an augmented linear system with the matrix denoted by $\mathcal{M}_{O}$,

$$
\mathcal{M}_{O}\left(\begin{array}{c}
\Phi_{h} \\
\bar{P}_{h}
\end{array}\right)=\left(\begin{array}{c}
F_{h} \\
0
\end{array}\right), \quad \mathcal{M}_{O}=\left(\begin{array}{cc}
\bar{A} & B \\
B^{T} & 0
\end{array}\right),
$$

This matrix sparsity pattern is represented on Fig. 5 . If $N_{x} \times N_{z}$ denotes the number of cells of the mesh, the block-matrix $\bar{A}$ denotes the finite element discretization of the integro-differential operator applied to $\phi^{\prime}$ on the left hand side of the equation (55), $\bar{A} \in \mathbb{R}^{N_{x}\left(N_{z}+2\right) \times N_{x}\left(N_{z}+2\right)}, B \in \mathbb{R}^{N_{x} \times N_{x}\left(N_{z}+2\right)}$ being the discretization of the Lagrangian contribution in the system matrix, $\left(\Phi_{h}, F_{h}\right) \in \mathbb{R}^{N_{x}\left(N_{z}+2\right)} \times \mathbb{R}^{N_{x}\left(N_{z}+2\right)}$ and $P_{h} \in \mathbb{R}^{N_{x}}$ denoting the vectors associated to $\phi^{\prime}$, the right hand side of the system and to the Lagrangian $\bar{P}$.

The integral discretization in the fluctuation equation (the last term of the left hand side) induces a fill-in of the system matrix, with a negative impact on the numerical method efficiency with respect to the memory requirements and the computational time. To improve the efficiency of the method a, this term is removed from the fluctuation equation using Eq. (52). This provides a second 
reformulation, proposed in [11], in which the equation (53) is substituted by

$$
\left\{\begin{array}{l}
-\varepsilon \frac{\partial}{\partial x}\left(A_{\perp} \frac{\partial \phi^{\prime}}{\partial x}\right)-\frac{\partial}{\partial z}\left(A_{\|} \frac{\partial \phi^{\prime}}{\partial z}\right)=\varepsilon f+\varepsilon \frac{\partial}{\partial x}\left(A_{\perp} \frac{\partial \bar{\phi}}{\partial x}\right), \\
\partial_{z} \phi^{\prime}=0 \quad \text { on } \Omega_{x} \times \Gamma_{z}, \\
\phi^{\prime}=0 \quad \text { on } \Gamma_{x} \times \Omega_{z}, \quad \overline{\phi^{\prime}}=0 \quad \text { in } \Omega_{x} .
\end{array}\right.
$$

The matrix obtained after a FEM discretization of the system (52),(56) gives rise to the linear system

$$
\left(\begin{array}{ccc}
A & \varepsilon C & B \\
\varepsilon C^{T} & \varepsilon A_{2} & 0 \\
B^{T} & 0 & 0
\end{array}\right)\left(\begin{array}{c}
\Phi_{h} \\
\bar{\Phi}_{h} \\
\bar{P}_{h}
\end{array}\right)=\left(\begin{array}{c}
F_{1} \\
F_{2} \\
0
\end{array}\right),
$$

$A \in \mathbb{R}^{N_{x}\left(N_{z}+2\right) \times N_{x}\left(N_{z}+2\right)}$ being the matrix discretizing the singular perturbation problem (48), $A_{2} \in \mathbb{R}^{N_{x} \times N_{x}}$ the one obtained after the discretization of the mean part equation (51), $C \in \mathbb{R}^{N_{x} \times N_{x}\left(N_{z}+2\right)}$ being the coupling term with the fluctuation. Finally $\bar{\Phi}_{h} \in \mathbb{R}^{N_{x}}$ is the vector associated with the mean part, $\left(F_{1}, F_{2}\right) \in \mathbb{R}^{N_{x}\left(N_{z}+2\right)} \times \mathbb{R}^{N_{x}}$ define the right hand side of the system. The plots of figure 5 show the benefits of this modified formulation, in which the equation for the fluctuation $\phi^{\prime}$ does not involve any integral operator. On a $500 \times 500$ mesh the number of non zeros elements stored in the matrix discretizing the equation (53) is 168 times larger than that of Problem (48), this ratio increasing further with mesh sizes [11]. In contrast, for Problem (52),(56) the non zeros elements remain 2.3 times larger that of Problem (48) whatever the mesh size.

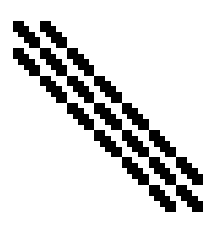

(a) $\mathcal{M}_{1}=A$

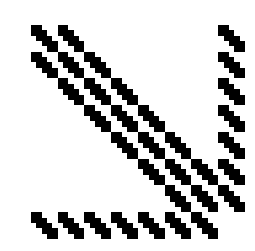

(b) $\mathcal{M}_{2}$

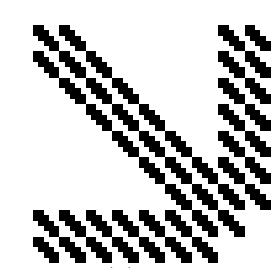

(c) $\mathcal{M}_{3}$

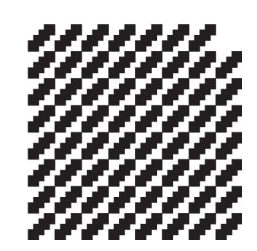

(d) $\mathcal{M}_{O}$

\begin{tabular}{|c|c|c|}
\hline Mat. & $\mathcal{M}_{1}=A$ & $\mathcal{M}_{2}=\left(\begin{array}{cc}A & B \\
B^{T} & 0\end{array}\right)$ \\
\hline \# rows & $N_{x}\left(N_{z}+2\right)$ & $N_{x}\left(N_{z}+3\right)$ \\
\hline Nnz & $\left(3 N_{z}+4\right)\left(3 N_{x}-2\right)$ & $\left(5 N_{z}+8\right)\left(3 N_{x}-2\right)$ \\
\hline \hline Mat. & $\mathcal{M}_{3}=\left(\begin{array}{ccc}A & \varepsilon C & B \\
\varepsilon C^{T} & \varepsilon A_{2} & 0 \\
B^{T} & 0 & 0\end{array}\right)$ & $\mathcal{M}_{O}=\left(\begin{array}{cc}\bar{A} & B \\
B^{T} & 0\end{array}\right)$ \\
\hline \# rows & $N_{x}\left(N_{z}+4\right)$ & $N_{x}\left(N_{z}+3\right)$ \\
\hline Nnz & $\left(7 N_{z}+13\right)\left(3 N_{x}-2\right)$ & $\left(N_{z}^{2}+6 N_{z}+8\right)\left(3 N_{x}-2\right)$ \\
\hline
\end{tabular}

Figure 5: Matrices obtained thanks to a $\mathbb{Q}_{1}-\mathrm{FEM}$ discretization of (a) the singular perturbation problem (48), (b) the fluctuation equation (56), (c) the AP formulation (52), (56), (d) the original fluctuation equation (53): plots of the structure pattern for a grid $\left(N_{x}, N_{z}\right)=(5,5)$ cells, matrix number of rows (\# rows) and number of non zeros elements (Nnz). 


\subsection{Solution via the primitive}

Another approach is proposed in [24] implementing the following characterization of the fluctuation space

$$
\begin{array}{r}
\psi^{\prime} \in \mathcal{A} \Longleftrightarrow \exists \chi \in \mathcal{W} \mid \psi^{\prime}=\partial_{z} \chi \quad \text { with } \\
\mathcal{W}:=\left\{\eta \in L^{2}(\Omega) \mid \partial_{z z}^{2} \eta \in L^{2}(\Omega), \partial_{x z}^{2} \eta \in L^{2}(\Omega), \eta=0 \text { on } \partial \Omega\right\},
\end{array}
$$

with $\Omega=\Omega_{x} \times \Omega_{z}$, the equation (56) can thus be recast into

Find $\chi \in \mathcal{W}$ such that

$$
\left(A_{\|} \partial_{z z}^{2} \chi, \partial_{z z}^{2} \eta\right)+\varepsilon\left(A_{\perp} \partial_{x z}^{2} \chi, \partial_{x z}^{2} \eta\right)=\varepsilon\left(f, \partial_{z} \eta\right)-\varepsilon\left(A_{\perp} \partial_{x} \bar{\phi}, \partial_{x z}^{2} \eta\right), \quad \forall \eta \in \mathcal{W},
$$

For homogeneous coefficients $A_{\perp}$, a strong formulation of the problem can be stated as

$$
\begin{cases}-\frac{\partial^{2}}{\partial z^{2}}\left(A_{\|} \frac{\partial^{2} \chi}{\partial z^{2}}\right)-\varepsilon A_{\perp} \frac{\partial^{2}}{\partial x^{2}}\left(\frac{\partial^{2} \chi}{\partial z^{2}}\right)=\varepsilon \frac{\partial \widetilde{f}}{\partial z}, & \text { in } \Omega, \\ \chi=0, & \text { on } \partial \Omega,\end{cases}
$$

with $\tilde{f}=f+\frac{\partial}{\partial x}\left(A_{\perp} \frac{\partial \bar{\phi}}{\partial x}\right)$. This system is transformed into two nested elliptic problems for $\phi^{\prime}=\partial \xi / \partial z$

$$
\begin{aligned}
& -\frac{\partial^{2}}{\partial z^{2}}\left(A_{\|} \zeta\right)-\varepsilon A_{\perp} \frac{\partial^{2} \zeta}{\partial x^{2}}=\varepsilon \frac{\partial \tilde{f}}{\partial z}, \quad \text { in } \Omega, \quad \zeta=0, \quad \text { on } \partial \Omega \\
& -\frac{\partial^{2} \xi}{\partial z^{2}}=-\zeta, \quad \text { in } \Omega, \quad \xi=0, \quad \text { on } \Gamma_{z} \times \Omega_{x} .
\end{aligned}
$$

The system (59b) is well posed and does not depend on $\varepsilon$. Moreover, in the problem (59a), the dominant operator in the limit $\varepsilon \rightarrow 0$ is supplemented with Dirichlet boundary conditions. Its kernel is thus reduced to zero. These two properties define a well posed problem for all $\varepsilon$. The implementation realized in [24] for anisotropic diffusion equations (with $A_{\perp}=0$ ) show that the numerical method is AP (see figure 6) providing computations with a precision independent of $\varepsilon$.

The advantage of this approach is to allow the resolution of the anisotropic problem by means of three standard elliptic problems, two nested elliptic problems for the fluctuation and one for the mean part, for which very efficient and proven methods exist. The recast of the fourth order problem (58) into two nested elliptic problems is straightforward for Neumann boundary conditions. However, the generalization to other kinds of boundary conditions and non homogeneous perpendicular coefficients remains to be done.

\subsection{Generalization to non adapted coordinates}

The generalization of the methods introduced in the preceding sections to anisotropy directions not aligned with one coordinate is documented in this 


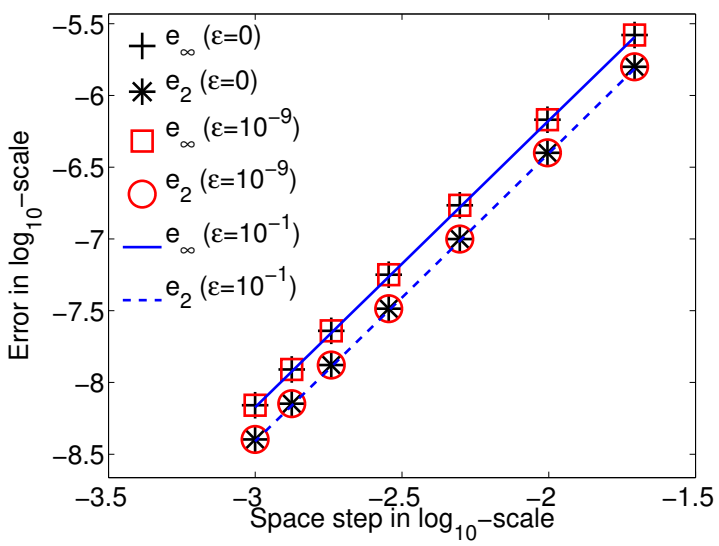

Figure 6: Relative error ( $L^{2}$ and $L^{\infty}$ norms) between the exact solution and the numerical approximation carried out thanks to "Solution via the primitive" of the fluctuation space, as a function of the mesh size and, for different anisotropy strengths [24].

section. The singular perturbation problem (48) is recast into

$$
\begin{cases}-\nabla_{\perp} \cdot\left(A_{\perp} \nabla \phi^{\varepsilon}\right)-\frac{1}{\varepsilon} \nabla_{\|} \cdot\left(A_{\|} \nabla_{\|} \phi^{\varepsilon}\right)=f^{\varepsilon}, & \text { in } \Omega, \\ \frac{1}{\varepsilon} n_{\|} \cdot \nabla_{\|} \phi^{\varepsilon}+n_{\perp} \cdot \nabla_{\perp} \phi^{\varepsilon}=0, & \text { on } \Gamma_{N}, \\ \phi^{\varepsilon}=0, & \text { on } \Gamma_{D} .\end{cases}
$$

with for $v \in \mathbb{R}^{3}, v_{\|}:=(b \cdot v) b, v_{\perp}:=(\mathbb{I} d-b \otimes b) v$, and $\nabla_{\|} \cdot v:=\nabla \cdot v_{\|}, \nabla_{\perp} \cdot v:=$ $\nabla \cdot v_{\perp}$, where $b$ is the unit vector pointing in the direction of the magnetic field $b=B /|B|$ (see equation (32)). The domain boundary $\Gamma=\partial \Omega, \Omega=\Omega_{x} \times \Omega_{z}$ is decomposed into $\Gamma=\Gamma_{N} \cup \Gamma_{D}$ with $\Gamma_{D}=\{x \in \Gamma, b(x) \cdot n(x)=0\}, n(x)$ being the outward normal.

The method implementing "Solution via the primitive" is straightforwardly extended to this framework. This approach has been genuinely developed for non adapted coordinates [23, 22]. More details are available in [24]. The plots of Fig. 7 report the precision of the numerical method as a function of the parameter $\alpha$ defined as the angle of the vector $b$ with the horizontal axis. From these computations, the precision of the method is observed to be almost independent of the anisotropy orientation, with a variation in the error norm lower than $10 \%$.

The extension of the duality based formulation is more intricate. Indeed, when one of the coordinates is not aligned with the anisotropy direction, the functional space for the mean value along the anisotropy direction is not easily discretized. The first generalization proposed in [50] implements a computation of the mean by duality. Introducing $\mathcal{V}:=\left\{\phi \in H^{1}(\Omega) \mid \phi=0\right.$ on $\left.\Gamma_{D}\right\}$ and the 


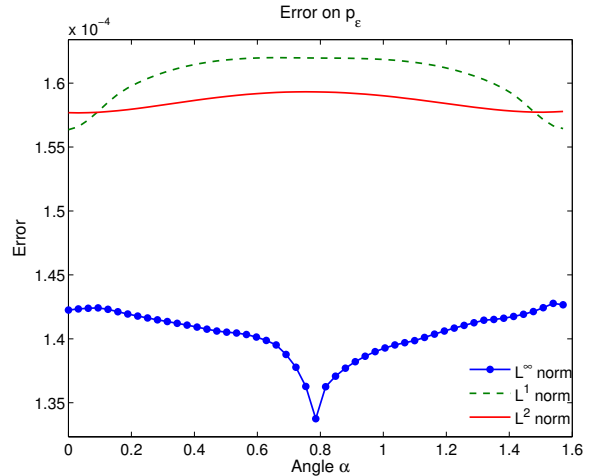

(a) $\left\|p_{\varepsilon}-p_{\varepsilon, a p p}\right\|_{\ell p} /\left\|p_{\varepsilon}\right\|_{\ell p}, \varepsilon=10^{-3}$.

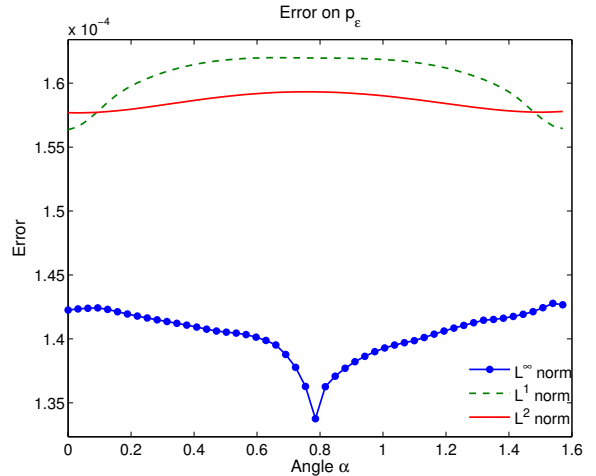

(b) $\left\|p_{\varepsilon}-p_{\varepsilon, a p p}\right\|_{\ell^{p}} /\left\|p_{\varepsilon}\right\|_{\ell^{p}}, \varepsilon=10^{-8}$.

Figure 7: Numerical implementation of the "Solution via the primitive" with anisotropies non aligned with one coordinate: Relative error between the exact solution $p_{\varepsilon}$ and the numerical approximation $p_{\varepsilon, a p p}$ as a function of the $\alpha$ the angle measured between the magnetic field direction and the $x$-axis for different anisotropy strength $\varepsilon=10^{-3}$ (left) and $\varepsilon=10^{-8}$ (right).

space of the mean functions $\mathcal{G}:=\left\{\psi \in \mathcal{V} \mid \nabla_{\|} \psi=0\right\}$, the space $\mathcal{A}$ of the fluctuations is defined by duality

$$
\mathcal{A}:=\{\varphi \in \mathcal{V} \mid(\varphi, \psi)=0, \quad \forall \psi \in \mathcal{G}\},
$$

using the orthogonal decomposition $\mathcal{V}=\mathcal{G} \otimes^{\perp} \mathcal{A}$, the $L^{2}$-scalar product being $(f, g):=\int_{\Omega} f g d x$.

With these definitions, the weak formulation of the singular perturbation problem (60) writes

Find $\left(p^{\varepsilon}, q^{\varepsilon}\right) \in \mathcal{G} \times \mathcal{A}$, such that

$$
\begin{cases}\left(A_{\perp} \nabla_{\perp} p^{\varepsilon}, \nabla_{\perp} \eta\right)+\left(A_{\perp} \nabla_{\perp} q^{\varepsilon}, \nabla_{\perp} \eta\right)=(f, \eta), & \forall \eta \in \mathcal{G}, \\ \left(A_{\|} \nabla_{\|} q^{\varepsilon}, \nabla_{\|} \xi\right)+\varepsilon\left(A_{\perp} \nabla_{\perp}\left(q^{\varepsilon}+p^{\varepsilon}\right), \nabla_{\perp} \xi\right)=(f, \xi), & \forall \xi \in \mathcal{A}\end{cases}
$$

Following the methodology used to derive the system (55), the discretization of the functional space $\mathcal{A}$ can be avoided thanks to the introduction of a Lagrange multiplier. This gives rise to the equivalent formulation

Find $\left(p^{\varepsilon}, q^{\varepsilon}, l^{\varepsilon}\right) \in \mathcal{G} \times \mathcal{V} \times \mathcal{G}$, such that

$$
\begin{cases}\left(A_{\perp} \nabla_{\perp} p^{\varepsilon}, \nabla_{\perp} \eta\right)+\left(A_{\perp} \nabla_{\perp} q^{\varepsilon}, \nabla_{\perp} \eta\right)=(f, \eta), & \forall \eta \in \mathcal{G}, \\ \left(A_{\|} \nabla_{\|} q^{\varepsilon}, \nabla_{\|} \xi\right)+\varepsilon\left(A_{\perp} \nabla_{\perp}\left(q^{\varepsilon}+p^{\varepsilon}\right), \nabla_{\perp} \xi\right)+\left(l^{\varepsilon}, \xi\right)=(f, \xi), & \forall \xi \in \mathcal{V}, \\ \left(q^{\varepsilon}, \chi\right)=0, & \forall \chi \in \mathcal{G},\end{cases}
$$

where the fluctuations are functions of the non constrained space $\mathcal{V}$, easily discretized by standard numerical methods. This feature comes at the price of an additional unknown, namely $l^{\varepsilon} \in \mathcal{G}$ penalizing the property $q^{\varepsilon} \in \mathcal{A}$. 
Finally, the same methodology is harnessed to penalize the property $\eta \in \mathcal{G}$ and $\chi \in \mathcal{G}$. To this end, the functional space of Lagrange multipliers $\mathcal{L}$ is introduced:

$\mathcal{L}:=\left\{\lambda \in L^{2}(\Omega) \mid \nabla_{\|} \lambda \in L^{2}(\Omega), \lambda=0\right.$ on $\left.\Gamma_{\text {in }}\right\}$, with $\Gamma_{\text {in }}:=\{x \in \Gamma \mid b(x) \cdot n(x)<0\}$

such that the weak formulation of the problem can be stated as

Find $\left(p^{\varepsilon}, q^{\varepsilon}, l^{\varepsilon}, \lambda^{\varepsilon}, \mu^{\varepsilon}\right) \in \mathcal{V} \times \mathcal{V} \times \mathcal{V} \times \mathcal{L} \times \mathcal{L}$, such that

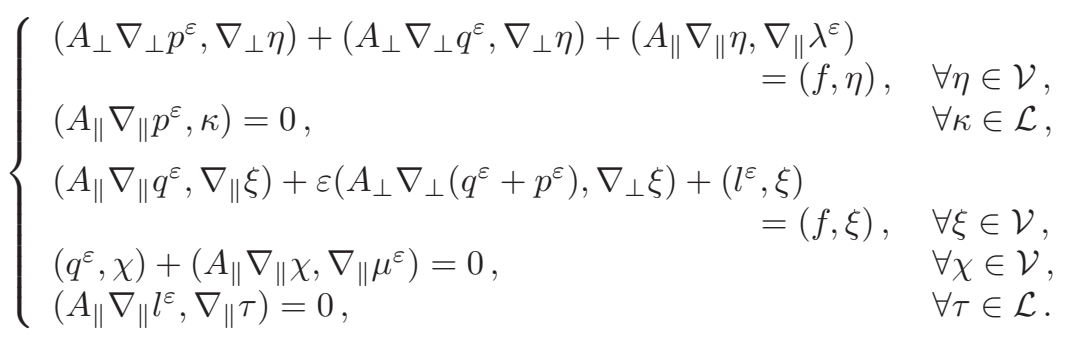

In this weak formulation of the singular perturbation problem, the discretization of the functional spaces $\mathcal{A}$ and $\mathcal{G}$ is not necessary. This is obtained thanks to the introduction of three auxiliary variables, namely $l^{\varepsilon}$ penalizing the constraint $q^{\varepsilon} \in \mathcal{A}$, as well as $\lambda^{\varepsilon}$ and $\mu^{\varepsilon}$ for the constraints $\eta \in \mathcal{G}$ and $\chi \in \mathcal{G}$.

Another route is proposed in [62] operating a "Micro-Macro" decomposition of the solution. It consists in splitting $\phi^{\varepsilon}$ into two non orthogonal components: $\phi^{\varepsilon}=p^{\varepsilon}+\varepsilon \mathfrak{q}^{\varepsilon}$, with $\mathfrak{q}^{\varepsilon} \in \mathcal{L}$. The fluctuation space $\mathcal{A}$ is replaced by a space populated with functions vanishing on one part of the boundary. This space is readily discretized by standard numerical methods. On top of that, the problem is formulated for the unknowns $\left(\phi^{\varepsilon}, \mathfrak{q}^{\varepsilon}\right)$, so that the discretization of the functional spaces is straightforward. The weak formulation of the problem is thus

Find $\left(\phi^{\varepsilon}, \mathfrak{q}^{\varepsilon}\right) \in \mathcal{V} \times \mathcal{L}$, such that

$$
\begin{cases}\left(A_{\perp} \nabla_{\perp} \phi^{\varepsilon}, \nabla_{\perp} v\right)+\left(A_{\|} \nabla_{\|} \mathfrak{q}^{\varepsilon}, \nabla_{\|} v\right)=(f, v), & \forall v \in \mathcal{V}, \\ \left(A_{\|} \nabla_{\|} \phi^{\varepsilon}, \nabla_{\|} w\right)=\varepsilon\left(A_{\|} \nabla_{\|} \mathfrak{q}^{\varepsilon}, \nabla_{\|} w\right), & \forall w \in \mathcal{L} .\end{cases}
$$

This system is Asymptotic-Preserving. Indeed, letting $\varepsilon \rightarrow 0$, the limit problem is recovered with

$$
\begin{aligned}
& \text { Find }\left(\phi^{0}, \mathfrak{q}^{0}\right) \in \mathcal{V} \times \mathcal{L}, \text { such that } \\
& \begin{cases}\left(A_{\perp} \nabla_{\perp} \phi^{0}, \nabla_{\perp} v\right)+\left(A_{\|} \nabla_{\|} \mathfrak{q}^{0}, \nabla_{\|} v\right)=(f, v), & \forall v \in \mathcal{V}, \\
\left(A_{\|} \nabla_{\|} \phi^{0}, \nabla_{\|} w\right)=0, & \forall w \in \mathcal{L} .\end{cases}
\end{aligned}
$$

In this formulation $\mathfrak{q}^{0}$ is the Lagrangian associated to the constraint $\nabla_{\|} \phi^{0}=0$.

Both methods provide the same Asymptotic-Preserving properties with coordinates and meshes not related to the anisotropy. They offer the same precision 
Table 2: Comparison of of the Micro-Macro (M.-M.) and Duality Based (D. B.) methods with a FEM discretization of the singular perturbation problem $(\mathrm{P})$ for coordinates non aligned to the anisotropy direction: number of rows, number of non zeros elements in the system matrix, computational time for the linear system resolution (thanks to the sparse direct solver MUMPS [1,2]) relative to that of the singular perturbation problem and $L^{2}$-error norm. The computations are carried out on a $100 \times 100$ mesh with a $\mathbb{Q}_{2}$ finite element method and $\varepsilon=10^{-6}$.

\begin{tabular}{|c||c|r|r||c|}
\hline Meth. & \# rows & \# Nnz & \multicolumn{1}{|c|}{ Time } & $L^{2}$-error \\
\hline \hline (M.-M.) & $20 \times 10^{3}$ & $623 \times 10^{3}$ & $231 \%$ & $3.34 \times 10^{-4}$ \\
\hline (D. B. $)$ & $50 \times 10^{3}$ & $1563 \times 10^{3}$ & $1478 \%$ & $3.34 \times 10^{-4}$ \\
\hline (P) & $10 \times 10^{3}$ & $156 \times 10^{3}$ & $100 \%$ & $3.27 \times 10^{-4}$ \\
\hline
\end{tabular}

but not at the same computational cost. To highlight their properties, the efficiency of the Micro-Macro and the Duality based formulations is compared, in table 2, to a standard FEM discretization of the singular perturbation problem for two dimensional computations. This later entry is aimed at providing a reference in term of memory and computational effort required to solve a standard elliptic problem. However, standards FEM discretizations of the singular perturbation problem only provides accurate solutions for moderate anisotropies while both AP methods are unaffected by the anisotropy strength. The introduction of the three Lagrangians increases significantly both the system matrix size and the number of non zero elements. The Micro-Macro scheme is much more efficient with a matrix size only twice as big as that of the standard method and a computational time increasing roughly to the same extent.

\subsection{Related works}

The versatility if the methods has been improved by developing the MicroMacro as well as the Duality-based methods to heterogeneous anisotropy ratios [62] with application to ionospheric plasma physics [11]. The Micro-Macro approach has been extended to closed magnetic field lines thanks to a regularization of the problem introduced in [121] and also implemented in [65] in the frame of resistive reduced MHD (see figure 8). Note that, an asymptotic preserving method based on a Lagrangian integration along the anisotropy direction is proposed in [29].

The efficiency of the Duality-based method has been significantly improved thanks to an hybrid method coupling the AP reformulation (52),(56) and the limit problem [51,39]. The development of this method is motivated by the results displayed on Figs. 4(b) and 4(c). The numerical approximation computed by either the limit problem (50) or an AP discretization have the same precision for small $\varepsilon$-values: the modelling error due to the use of $\left(P^{0}\right)$ for $\varepsilon>0$ may be negligible compared to the discretization error. However, the numerical resolution of the limit model is much more efficient in term of computational efforts, since the solutions of $P^{0}$ do not depend on $z$. The hybrid method relies on a domain decomposition, using an AP scheme for the sub-domain with the 

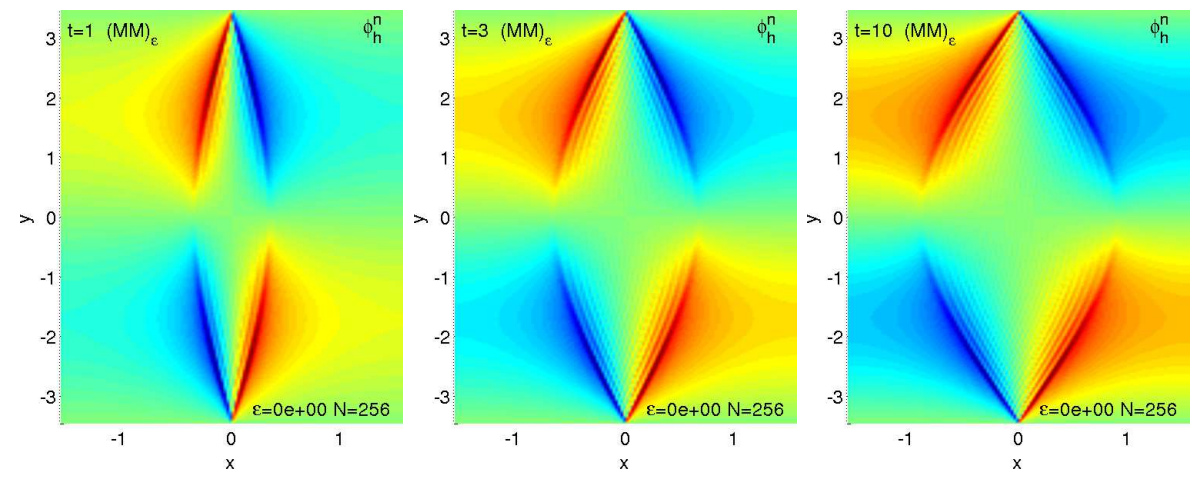

Figure 8: Evolution of a magnetic island simulated by an Asymptotic-Preserving discretization of the resistive reduced Magneto-Hydro-Dynamic implementing the stabilized Micro-Macro method. Plots of the stream function carried out on a mesh with $N_{x} \times N_{y}=256 \times 256$ cells (see [65]).

largest values of $\varepsilon$ and the limit model elsewhere. It is thus all more efficient than the sub-domain on which the limit model is used can be enlarged. Table 3 provides some elements relating the gain brought by this coupling strategy. As

Table 3: Efficiency of the hybrid (HYB.) and the Asymptotic-Preserving (AP) methods compared to a FEM discretization of the problem $(48)\left(P^{\varepsilon}\right)$ : Number of cells of the grid $\left(N=N_{x}=N_{z}\right)$, resolution time (with the sparse direct solver MUMPS [1]) relative to that of the singular perturbation problem (48) $(\mathrm{T})$, number of non zero elements stored in the factorized matrix (Nnz fact.), number of rows (\# rows), number of non zero elements stored in the system matrix (Nnz Mat.) and precision of the numerical approximation $\left(\mathrm{L}^{2}\right.$-error norm of the relative error), for $\Omega_{z}=\Omega_{z}^{1} \cup \Omega_{z}^{2}$, where $\operatorname{mes}\left(\Omega_{z}^{2}\right)=7 / 10 \operatorname{mes}\left(\Omega_{z}\right), \Omega_{z}^{2}$ being the sub-domain of the limit problem [39].

\begin{tabular}{|c|c|r|r|r|r|r|}
\hline Met. & \multicolumn{1}{|c|}{$N$} & \multicolumn{1}{c|}{ T } & \multicolumn{1}{|c|}{ Nnz (fact.) } & \multicolumn{1}{|c|}{ \#rows } & Nnz (Mat.) & error $\left(\mathrm{L}^{2}\right)$ \\
\hline \hline (HYB.) & 500 & $53 \%$ & 9861960 & 77000 & 1592374 & $1.4 \times 10^{-5}$ \\
\hline$(\mathrm{AP})$ & 500 & $203 \%$ & 40655248 & 252000 & 5262474 & $1.4 \times 10^{-5}$ \\
\hline$\left(P^{\varepsilon}\right)$ & 500 & $100 \%$ & 26940422 & 251000 & 2252992 & $3.4 \times 10^{-3}$ \\
\hline \hline$($ HYB. $)$ & 2000 & $26 \%$ & 206531976 & 1208000 & 25269574 & $1.2 \times 10^{-6}$ \\
\hline$(\mathrm{AP})$ & 2000 & $137 \%$ & 804867106 & 4008000 & 84049974 & $8.8 \times 10^{-7}$ \\
\hline$\left(P^{\varepsilon}\right)$ & 2000 & $100 \%$ & 557859738 & 4004000 & 36011992 & $1.5 \times 10^{-2}$ \\
\hline
\end{tabular}

mentioned above, the overhead of the AP method compared to the numerical resolution of the singular perturbation problem, with respect to the memory requirements is roughly 2.3 for the most refined meshes. The fill-in of the factorized matrix does not scale as badly, with an increase of the number of non zero elements stored from $50 \%$ to $80 \%$. When the limit problem is discretized on a large enough sub-domain, the fill-in of the hybrid method is lower than that of the singular problem (48) with a computational time necessary for the 
linear system resolution being divided by 3 .

\section{Conclusions}

In this document, Asymptotic-Preserving methods are reviewed in the frame of three singular perturbation problems. First the concept of AsymptoticPreserving methods are outlined for the quasi-neutral limit of plasma descriptions. The scale of interest here is the Debye length measuring the typical size of the space charge creations. In this context, the derived AP-schemes offer the possibility to choose the model harnessed accordingly to the needs of the physics. Indeed, when the discretization parameters are large compared to the Debye length, AP-schemes define a consistent discretization of a quasi-neutral model, with properties similar to Magneto-Hydro-Dynamic descriptions (however with a finite electron inertia and eventually a kinetic description). Conversely, local up-scaling are possible, by adjusting the mesh size to the local Debye length, the numerical methods becoming consistent with the non quasi-neutral model (either the Vlasov-Maxwell or the Euler-Maxwell system depending on the description used for the plasma, electrostatic models being also proposed).

The systematic methodology implemented to derive AP scheme is decomposed in different steps. First the limit regime needs to be clearly identified. It consists of a set of equations providing the limit of the solution in the asymptotic regime. In the framework of singular perturbation problem, this set of equations is not readily obtained from the initial multiscale problem. The asymptotic analysis is a crucial tool to clarify the inter relations of the multiscale and the limit problems. More importantly, the derivation of the limit (quasi-neutral) problem from the multiscale (non quasi-neutral) set of equations is a key point in the construction of Asymptotic-Preserving methods. This preliminary work is thus capitalized on to manufacture a set of reformulated equations in which the quasi-neutral limit is regular: the limit problem is recovered by setting formally the asymptotic parameter to zero in the reformulated system. In this specific context, the asymptotic parameter is defined as the ratio of the Debye length and the typical length of interest, or equivalently the mesh size. Until this stage all the analysis are free from any numerical method. Finally, the question of the discretization is addressed. Performing the same analysis but with discretized equations, allows to derive an asymptotic preserving numerical method.

Two other frameworks are also addressed in the present document. The first one is related to the drift regime for fluid description of tokamak plasmas, also referred to as the gyro-fluid limit. The asymptotic regime investigated is the limit of infinite acoustic wave speeds (low Mach regime) with local transitions to flows characterized by Mach numbers close to one. In the low Mach regime, the parallel momentum equation degenerates into an equilibrium relation, with a pressure gradient balancing the electric force. No contribution of the parallel momentum appears explicitly in this equation anymore, which is at the origin of the singular nature of the drift limit. The applications envisioned here are more specifically related to the tokamak plasma edge physics, with a different dynamic in the plasma core compared to that of the sheath created in the 
vicinity of the wall. The derivation of asymptotic preserving schemes, in both the frameworks of the quasi-neutral and the drift regimes, involves the resolution of anisotropic elliptic or diffusion problems. This class of equations define a singular perturbation problem when the boundary conditions supplementing the diffusion operator in the aligned direction (with respect to the magnetic field) translate, for instance, the periodicity of the torus. Finally, section 4 is dedicated to the last framework dedicated to asymptotic preserving methods developed to address efficiently these problems.

The systematic derivation of AP-methods can be extended to more singular limits. Some examples can be named, with an extension of the quasi-neutral limit investigations aiming to bridge the Vlasov-Maxwell system and a MagnetoHydro-Dynamic model or the Vlasov-Poisson system and the Boltzmann relation for the electrons. The adiabatic response as well as MHD models are successfully operated for the simulation, because they filter out from the equations most of the high frequencies and give access to the macroscopic evolution in an efficient way. In both cases the limit is much more singular, since in addition to the quasineutral asymptotic, a fluid and a mass-less (for the electron) limits are necessary to define the reduced models. Another extension can be considered in the gyrofluid framework, with the investigation of the quasi-neutrality break down, in order to address extensively the regimes transition between the plasma core and the sheath. A more distant issue can also be envisioned, with the extension of AP-methods to other scientific fields where multiscale problems are common, namely biology and complex systems for instance, where this class of methods, if already investigated, lacks significant developments.

\section{Acknowledgments}

This work has been carried out within the framework of the EUROfusion Consortium and has received funding from the Euratom research and training programme 2014-2018 under grant agreement No 633053. The views and opinions expressed herein do not necessarily reflect those of the European Commission.

Furthermore, FD would like to acknowledge support from the ANR MOONRISE (MOdels, Oscillations and NumeRIcal SchEmes, 2015-2019) and partial support from ANR PEPPSI (Plasma Edge Physics and Plasma-Surface Interactions, 2013-2017). This work has been supported by the National Science Foundation (NSF) under grant RNMS11-07444 (KI-Net). PD is on leave from CNRS, Institut de Mathématiques de Toulouse, France. PD acknowledges support from the Royal Society and the Wolfson foundation through a Royal Society Wolfson Research Merit Award.

[1] P. R. Amestoy, I. S. Duff, J.-Y. L'Excellent, and J. Koster. A fully asynchronous multifrontal solver using distributed dynamic scheduling. SIAM Journal on Matrix Analysis and Applications, 23(1):15-41, Jan. 2001.

[2] P. R. Amestoy, A. Guermouche, and S. Pralet. Hybrid scheduling for the parallel solution of linear systems. Parallel Computing, 32:136-156, 2006. 
[3] A. A. Amsden and F. H. Harlow. Transport of turbulence in numerical fluid dynamics. Journal of Computational Physics, 3(1):94-110, 1968.

[4] R. Balescu. Transport Processes in Plasmas: Neoclassical Transport Theory. North-Holland, 1988.

[5] R. Barthelmé, P. Ciarlet, Jr., and E. Sonnendrücker. Generalized formulations of Maxwell's equations for numerical Vlasov-Maxwell simulations. Math. Models Methods Appl. Sci., 17(5):657-680, 2007.

[6] M. A. Beer, S. C. Cowley, and G. W. Hammett. Field-aligned coordinates for nonlinear simulations of tokamak turbulence. Physics of Plasmas (1994-present), 2(7):2687-2700, July 1995.

[7] R. Belaouar, N. Crouseilles, P. Degond, and E. Sonnendrücker. An asymptotically stable semi-Lagrangian scheme in the quasi-neutral limit. J. Sci. Comput., 41(3):341-365, 2009.

[8] P. M. Bellan. Fundamentals of plasma physics. Cambridge University Press, Cambridge; New York, 2008.

[9] M. Bennoune, M. Lemou, and L. Mieussens. Uniformly stable numerical schemes for the Boltzmann equation preserving the compressible Navier-Stokes asymptotics. Journal of Computational Physics, 227(8):3781-3803, 2008.

[10] C. Besse, R. Carles, and F. Méhats. An Asymptotic Preserving Scheme Based on a New Formulation for NLS in the Semiclassical Limit. Multiscale Modeling E3 Simulation, 11(4):1228-1260, Jan. 2013.

[11] C. Besse, F. Deluzet, C. Negulescu, and C. Yang. Efficient Numerical Methods for Strongly Anisotropic Elliptic Equations. Journal of Scientific Computing, 55(1):231-254, Apr. 2013.

[12] Besse, C. and Claudel, J. and Degond, P. and Deluzet, F. and Gallice, G. and Tessieras, C. A model hierarchy for ionospheric plasma modeling. Mathematical Models and Methods in Applied Sciences, 14(3):393-416, 2004.

[13] M. Bessemoulin-Chatard, C. Chainais-Hillairet, and M. Vignal. Study of a Finite Volume Scheme for the Drift-Diffusion System. Asymptotic Behavior in the Quasi-Neutral Limit. SIAM Journal on Numerical Analysis, 52(4):1666-1691, Jan. 2014.

[14] C. Birdsall and A. Langdon. Plasma physics via computer simulation. Inst of Physics Pub Inc, 2004.

[15] D. Biskamp. Nonlinear Magnetohydrodynamics. Cambridge University Press, July 1997. 
[16] D. Biskamp. Magnetic Reconnection in Plasmas. Cambridge University Press, Sept. 2005.

[17] J. A. Bittencourt. Fundamentals of Plasma Physics. Springer, 3rd edition edition, Aug. 2004.

[18] J. P. Boris. Proceedings of the "4th Conference on Numerical Simulation of Plasmas". In Relativistic plasma simulation-optimization of a hybrid code, page pp.67. Naval Res. Lab., Washington, D.C., Nov. 1970.

[19] S. Boscarino, L. Pareschi, and G. Russo. Implicit-Explicit Runge-Kutta Schemes for Hyperbolic Systems and Kinetic Equations in the Diffusion Limit. SIAM Journal on Scientific Computing, 35(1):A22-A51, Jan. 2013.

[20] J. U. Brackbill and D. W. Forslund. An implicit method for electromagnetic plasma simulation in two dimensions. J. Comput. Phys., 46(2):271$308,1982$.

[21] S. I. Braginskii. Transport Processes in a Plasma. Reviews of Plasma Physics, 1:205, 1965.

[22] S. Brull, P. Degond, and F. Deluzet. Degenerate Anisotropic Elliptic Problems and Magnetized Plasma Simulations. Communications in Computational Physics, 11(1):147-178, 2012.

[23] S. Brull, P. Degond, F. Deluzet, and A. Mouton. Asymptotic-Preserving scheme for a bi-fluid Euler-Lorentz model. Kinetic and Related Models, 4(4):991-1023, Dec. 2011.

[24] S. Brull, F. Deluzet, and A. Mouton. Numerical resolution of an anisotropic non-linear diffusion problem. Communications in Mathematical Sciences, 13(1):203-224, 2015.

[25] H. Bufferand, G. Ciraolo, G. Dif-Pradalier, P. Ghendrih, P. Tamain, Y. Marandet, and E. Serre. Study of transition to supersonic regime in divertor SOL using SolEdge2d code. Plasma Physics and Controlled Fusion, 56(12):122001, 2014.

[26] J. A. Carrillo and B. Yan. An Asymptotic Preserving Scheme for the Diffusive Limit of Kinetic Systems for Chemotaxis. Multiscale Modeling ES Simulation, 11(1):336-361, Jan. 2013.

[27] C. Cercignani, V. Gerasimenko, and D. I. Petrina. Many-Particle Dynamics and Kinetic Equations. Springer Science \& Business Media, July 1997.

[28] C. Cercignani and D. H. Sattinger. Scaling Limits and Models in Physical Processes. Springer Science \& Business Media, Sept. 1998. 
[29] L. Chacón, D. del Castillo-Negrete, and C. D. Hauck. An asymptoticpreserving semi-Lagrangian algorithm for the time-dependent anisotropic heat transport equation. Journal of Computational Physics, 272:719-746, Sept. 2014.

[30] F. Chen. Introduction to plasma physics and controlled fusion: Plasma physics. Plenum Pub Corp, 1984.

[31] F. F. Chen. An Indispensable Truth: How Fusion Power Can Save the Planet. Springer, 1st edition. edition, Apr. 2011.

[32] G. Chen and L. Chacón. A multi-dimensional, energy- and chargeconserving, nonlinearly implicit, electromagnetic Vlasov-Darwin particlein-cell algorithm. Computer Physics Communications, 197:73-87, 2015.

[33] R. Chodura. Plasma Flow in the Sheath and the Presheath of a ScrapeOff Layer. In D. E. Post and R. Behrisch, editors, Physics of Plasma-Wall Interactions in Controlled Fusion, pages 99-134. Springer US, Jan. 1986.

[34] B. I. Cohen, A. Langdon, D. W. Hewett, and R. J. Procassini. Performance and optimization of direct implicit particle simulation. Journal of Computational Physics, 81(1):151 - 168, 1989.

[35] B. L. Cohen, A. B. Langdon, and A. Friedman. Implicit time integration for plasma simulation. J. Comput. Phys., 46(1):15-38, 1982.

[36] P. Colella and K. Pao. A Projection Method for Low Speed Flows. Journal of Computational Physics, 149(2):245-269, Mar. 1999.

[37] F. Cordier, P. Degond, and A. Kumbaro. An Asymptotic-Preserving allspeed scheme for the Euler and Navier-Stokes equations. Journal of Computational Physics, 231(17):5685-5704, 2012.

[38] A. Crestetto, N. Crouseilles, and M. Lemou. Kinetic/fluid micro-macro numerical schemes for Vlasov-Poisson-BGK equation using particles. Kinetic and Related Models, 5(4):787-816, Nov. 2012.

[39] A. Crestetto, F. Deluzet, and C. Negulescu. A Hybrid Method for Anisotropic Elliptic Problems Based on the Coupling of an AsymptoticPreserving Method with the Asymptotic Limit Model. SIAM Journal on Scientific Computing, 38(3):A1821-A1847, Jan. 2016.

[40] P. Crispel, P. Degond, and M.-H. Vignal. Quasi-neutral fluid models for current-carrying plasmas. Journal of Computational Physics, 205(2):408438, May 2005.

[41] P. Crispel, P. Degond, and M.-H. Vignal. An asymptotic preserving scheme for the two-fluid Euler-Poisson model in the quasineutral limit. J. Comput. Phys., 223(1):208-234, 2007. 
[42] P. Crispel, P. Degond, and M. H. Vignal. A plasma expansion model based on the full Euler-Poisson system. Mathematical Models and Methods in Applied Sciences, 17(07):1129-1158, July 2007.

[43] N. Crouseilles, G. Dimarco, and M. Vignal. Multiscale Schemes for the BGK-Vlasov-Poisson System in the Quasi-Neutral and Fluid Limits. Stability Analysis and First Order Schemes. Multiscale Modeling \& Simulation, pages 65-95, Jan. 2016.

[44] N. Crouseilles, M. Kuhn, and G. Latu. Comparison of Numerical Solvers for Anisotropic Diffusion Equations Arising in Plasma Physics. Journal of Scientific Computing, pages 1-38, Feb. 2015.

[45] N. Crouseilles and M. Lemou. An asymptotic preserving scheme based on a micro-macro decomposition for Collisional Vlasov equations: diffusion and high-field scaling limits. Kinetic and Related Models, 4(2):441-477, Apr. 2011.

[46] N. Crouseilles and G. Manfredi. Asymptotic preserving schemes for the Wigner-Poisson-BGK equations in the diffusion limit. Computer Physics Communications, 185(2):448-458, 2014.

[47] P. A. Davidson. An introduction to magnetohydrodynamics. Cambridge University Press, Cambridge, 2001.

[48] P. Degond. Panoramas et synthèses, N. 39-40: Numerical Models for Fusion, chapter 1, pages 1-90. SMF, Paris, France, 2013.

[49] P. Degond, F. Deluzet, and D. Doyen. Asymptotic-preserving ParticleIn-Cell methods for the Vlasov-Maxwell system near quasi-neutrality. arXiv:1509.04235 [physics] (Submitted to Journal of Computational Physics), Sept. 2015. arXiv: 1509.04235.

[50] P. Degond, F. Deluzet, A. Lozinski, J. Narski, and C. Negulescu. Dualitybased asymptotic-preserving method for highly anisotropic diffusion equations. Communications in Mathematical Sciences, 10(1):1-31, 2012.

[51] P. Degond, F. Deluzet, D. Maldarella, J. Narski, C. Negulescu, and M. Parisot. Hybrid model for the Coupling of an Asymptotic Preserving scheme with the Asymptotic Limit model: The One Dimensional Case. ESAIM: Proceedings, 32:23-30, Oct. 2011.

[52] P. Degond, F. Deluzet, and L. Navoret. An asymptotically stable Particlein-Cell (PIC) scheme for collisionless plasma simulations near quasineutrality. Comptes Rendus Mathematique, 343(9):613-618, Nov. 2006.

[53] P. Degond, F. Deluzet, L. Navoret, A.-B. Sun, and M.-H. Vignal. Asymptotic-Preserving Particle-In-Cell method for the Vlasov-Poisson system near quasineutrality. Journal of Computational Physics, 229(16):5630-5652, 2010. 
[54] P. Degond, F. Deluzet, and C. Negulescu. An Asymptotic Preserving Scheme for Strongly Anisotropic Elliptic Problems. Multiscale Modeling 6 Simulation, 8(2):645-666, 2010.

[55] P. Degond, F. Deluzet, A. Sangam, and M.-H. Vignal. An Asymptotic Preserving scheme for the Euler equations in a strong magnetic field. Journal of Computational Physics, 228(10):3540-3558, 2009.

[56] P. Degond, F. Deluzet, and D. Savelief. Numerical approximation of the Euler-Maxwell model in the quasineutral limit. Journal of Computational Physics, 231(4):1917-1946, 2012.

[57] P. Degond, V. Grandgirard, Y. Sarazin, S. C. Jardin, and C. Villani. Numerical models for fusion. Société Mathématique de France, 2013, Paris, France, 2013.

[58] P. Degond and S. Jin. A Smooth Transition Model between Kinetic and Diffusion Equations. SIAM Journal on Numerical Analysis, 42(6):26712687, Jan. 2005.

[59] P. Degond, S. Jin, and M. Tang. On the Time Splitting Spectral Method for the Complex Ginzburg-Landau Equation in the Large Time and Space Scale Limit. SIAM Journal on Scientific Computing, 30(5):2466-2487, Jan. 2008.

[60] P. Degond, S. Jin, and J. Yuming. Mach-number uniform asymptoticpreserving gauge schemes for compressible flows. Bulletin-Institute of Mathematics Academia Sinica, 2(4):851, 2007.

[61] P. Degond, H. Liu, D. Savelief, and M.-H. Vignal. Numerical Approximation of the Euler-Poisson-Boltzmann Model in the Quasineutral Limit. Journal of Scientific Computing, 51(1):59-86, Apr. 2012.

[62] P. Degond, A. Lozinski, J. Narski, and C. Negulescu. An asymptoticpreserving method for highly anisotropic elliptic equations based on a Micro-Macro decomposition. Journal of Computational Physics, 231(7):2724-2740, 2012.

[63] P. Degond and M. Tang. All Speed Scheme for the Low Mach Number Limit of the Isentropic Euler Equations. Communications in Computational Physics, 2011.

[64] D. del Castillo-Negrete and L. Chacón. Parallel heat transport in integrable and chaotic magnetic fields. Physics of Plasmas, 19(5):056112056112-13, Mar. 2012.

[65] F. Deluzet, C. Negulescu, M. Ottaviani, and S. Possanner. Numerical study of the plasma tearing instability on the resistive time scale. Journal of Computational Physics, 280:602-625, Jan. 2015. 
[66] F. Deluzet, S. Possanner, and M. Ottaviani. The drift limit in the EulerLorentz equations via Asymptotic-Preservig numerical simulations. Submitted to Journal of Computational Physics.

[67] G. Dimarco and L. Pareschi. Asymptotic preserving Implicit-Explicit Runge-Kutta methods for non linear kinetic equations. arXiv:1205.0882 [math-ph], May 2012.

[68] G. Dimarco, L. Pareschi, and V. Rispoli. Implicit-Explicit RungeKutta schemes for the Boltzmann-Poisson system for semiconductors. arXiv:1305.1759 [math-ph, physics:physics], May 2013.

[69] A. M. Dimits. Fluid simulations of tokamak turbulence in quasiballooning coordinates. Physical Review E, 48(5):4070-4079, Nov. 1993.

[70] A. M. Dimits, G. Bateman, M. A. Beer, B. I. Cohen, W. Dorland, G. W. Hammett, C. Kim, J. E. Kinsey, M. Kotschenreuther, A. H. Kritz, L. L. Lao, J. Mandrekas, W. M. Nevins, S. E. Parker, A. J. Redd, D. E. Shumaker, R. Sydora, and J. Weiland. Comparisons and physics basis of tokamak transport models and turbulence simulations. Physics of Plasmas, 7(3):969, 2000.

[71] B. D. Dudson, M. V. Umansky, X. Q. Xu, P. B. Snyder, and H. R. Wilson. BOUT ++: A framework for parallel plasma fluid simulations. Computer Physics Communications, 180(9):1467-1480, Sept. 2009.

[72] P. H. F Hariri. The flux-coordinate independent approach applied to Xpoint geometries. Physics of Plasmas, 2117(14), 2014.

[73] F. Filbet, C. Negulescu, and C. Yang. Numerical study of a nonlinear heat equation for plasma physics. Int. J. Comput. Math., 89(8):1060-1082, May 2012.

[74] R. Fitzpatrick. Plasma Physics: An Introduction. CRC Press, Boca Raton, Sept. 2014.

[75] J. P. Freidberg. Plasma Physics and Fusion Energy. Cambridge University Press, Cambridge, 2007.

[76] J. P. Freidberg. Ideal MHD. Cambridge University Press, Cambridge, 2014.

[77] X. Garbet, Y. Idomura, L. Villard, and T. H. Watanabe. Gyrokinetic simulations of turbulent transport. Nuclear Fusion, 50(4):043002, Mar. 2010 .

[78] A. V. Gordeev, A. S. Kingsep, and L. I. Rudakov. Electron magnetohydrodynamics. Physics Reports, 243(5):215-315, July 1994. 
[79] V. Grandgirard, M. Brunetti, P. Bertrand, N. Besse, X. Garbet, P. Ghendrih, G. Manfredi, Y. Sarazin, O. Sauter, E. Sonnendrücker, J. Vaclavik, and L. Villard. A drift-kinetic Semi-Lagrangian 4d code for ion turbulence simulation. Journal of Computational Physics, 217(2):395-423, Sept. 2006.

[80] S. Guisset, S. Brull, B. Dubroca, E. d'Humières, S. Karpov, and I. Potapenko. Asymptotic-Preserving Scheme for the M 1-Maxwell System in the Quasi-Neutral Regime. Communications in Computational Physics, 19(02):301-328, Feb. 2016.

[81] S. Günter, Q. Yu, J. Krüger, and K. Lackner. Modelling of heat transport in magnetised plasmas using non-aligned coordinates. J. Comput. Phys., 209(1):354-370, Oct. 2005.

[82] J. Haack, S. Jin, and J. Liu. An All-Speed Asymptotic-Preserving Method for the Isentropic Euler and Navier-Stokes Equations. Communications in Computational Physics, 12(04):955-980, Oct. 2012.

[83] G. W. Hammett, M. A. Beer, W. Dorland, S. C. Cowley, and S. A. Smith. Developments in the gyrofluid approach to Tokamak turbulence simulations. Plasma Physics and Controlled Fusion, 35(8):973, 1993.

[84] F. Hariri and M. Ottaviani. A flux-coordinate independent field-aligned approach to plasma turbulence simulations. Computer Physics Communications, 184(11):2419-2429, Nov. 2013.

[85] F. H. Harlow and A. A. Amsden. Numerical calculation of almost incompressible flow. Journal of Computational Physics, 3(1):80-93, 1968.

[86] R. D. Hazeltine and J. D. Meiss. Plasma Confinement. Dover Publications, Dec. 2003.

[87] R. D. Hazeltine and F. Waelbroeck. The framework of plasma physics. Westview Press, Boulder, CO, 2004.

[88] D. W. Hewett. Low-frequency electromagnetic (Darwin) applications in plasma simulation. Computer Physics Communications, 84(1-3):243-277, Nov. 1994.

[89] D. W. Hewett and A. B. Langdon. Electromagnetic direct implicit plasma simulation. Journal of Computational Physics, 72(1):121 - 155, 1987.

[90] P. Hill, F. Hariri, and M. Ottaviani. The effect of magnetic islands on Ion Temperature Gradient turbulence driven transport. Physics of Plasmas (1994-present), 22(4):042308, Apr. 2015.

[91] R. Hockney and J. Eastwood. Computer simulation using particles. Taylor \& Francis, 1988. 
[92] J. Hu, Q. Li, and L. Pareschi. Asymptotic-Preserving Exponential Methods for the Quantum Boltzmann Equation with High-Order Accuracy. Journal of Scientific Computing, 62(2):555-574, May 2014.

[93] J. D. Huba. NRL PLASMA FORMULARY. Technical report, Naval Research Lab., Washington, DC 20375, 2011.

[94] G. T. A. Huysmans, S. Pamela, E. van der Plas, and P. Ramet. Non-linear MHD simulations of edge localized modes (ELMs). Plasma Physics and Controlled Fusion, 51(12):124012, Dec. 2009.

[95] F. James and N. Vauchelet. Numerical Methods for One-Dimensional Aggregation Equations. SIAM Journal on Numerical Analysis, 53(2):895916, Jan. 2015.

[96] J. Jang, F. Li, J.-M. Qiu, and T. Xiong. High order asymptotic preserving DG-IMEX schemes for discrete-velocity kinetic equations in a diffusive scaling. Journal of Computational Physics, 281:199-224, Jan. 2015.

[97] S. Jardin. Review of implicit methods for the magnetohydrodynamic description of magnetically confined plasmas. Journal of Computational Physics, 231(3):822-838, 2012.

[98] S. Jin. Efficient Asymptotic-Preserving (AP) Schemes For Some Multiscale Kinetic Equations. SIAM Journal on Scientific Computing, 21:441454, Sept. 1999. ACM ID: 339309.

[99] S. Jin. Asymptotic preserving (AP) schemes for multiscale kinetic and hyperbolic equations: a review. Lecture Notes for Summer School on "Methods and Models of Kinetic Theory" June 2010, Rivista di Matematica della Universita di Parma, pages 177-206, 2012.

[100] S. Jin and C. D. Levermore. Numerical Schemes for Hyperbolic Conservation Laws with Stiff Relaxation Terms. Journal of Computational Physics, 126(2):449-467, 1996.

[101] S. Jolliet, A. Bottino, P. Angelino, R. Hatzky, T. M. Tran, B. F. Mcmillan, O. Sauter, K. Appert, Y. Idomura, and L. Villard. A global collisionless PIC code in magnetic coordinates. Computer Physics Communications, 177(5):409-425, Sept. 2007.

[102] G. Joyce, M. Lampe, S. P. Slinker, and W. M. Manheimer. Electrostatic particle-in-cell simulation technique for quasineutral plasma. Journal of Computational Physics, 138(2):540-562, 1997.

[103] N. A. Krall and A. W. Trivelpiece. Principles of Plasma Physics. San Francisco Press, Incorporated, June 1986.

[104] P. Lafitte, A. Lejon, and G. Samaey. A High-Order Asymptotic-Preserving Scheme for Kinetic Equations Using Projective Integration. SIAM Journal on Numerical Analysis, 54(1):1-33, Jan. 2016. 
[105] A. B. Langdon, B. I. Cohen, and A. Friedman. Direct implicit large timestep particle simulation of plasmas. J. Comput. Phys., 51(1):107-138, 1983.

[106] I. Langmuir. The interaction of electron and positive ion space charges in cathode sheaths. Physical Review, 33(6):954-989, 1929.

[107] G. Lapenta. Particle simulations of space weather. Journal of Computational Physics, 231(3):795-821, Feb. 2012.

[108] E. W. larsen and J. E. Morel. Asymptotic solutions of numerical transport problems in optically thick, diffusive regimes II. Journal of Computational Physics, 83(1):212-236, 1989.

[109] E. W. Larsen, J. E. Morel, and W. F. Miller. Asymptotic solutions of numerical transport problems in optically thick, diffusive regimes. Journal of Computational Physics, 69(2):283-324, 1987.

[110] R. G. Littlejohn. Variational principles of guiding centre motion. Journal of Plasma Physics, 29(01):111-125, Feb. 1983.

[111] J. Liu and L. Mieussens. Analysis of an Asymptotic Preserving Scheme for Linear Kinetic Equations in the Diffusion Limit. SIAM Journal on Numerical Analysis, 48(4):1474-1491, Jan. 2010.

[112] A. Lozinski, J. Narski, and C. Negulescu. Highly anisotropic temperature balance equation and its asymptotic-preserving resolution. arXiv:1203.6739 [math], Mar. 2012. arXiv: 1203.6739.

[113] H. Lütjens and J.-F. Luciani. The XTOR code for nonlinear 3d simulations of MHD instabilities in tokamak plasmas. Journal of Computational Physics, 227(14):6944-6966, July 2008.

[114] D. Maisonnier, D. Campbell, I. Cook, L. Di Pace, L. Giancarli, J. Hayward, A. Li Puma, M. Medrano, P. Norajitra, M. Roccella, P. Sardain, M. Tran, and D. Ward. Power plant conceptual studies in Europe. Nuclear Fusion, 47(11):1524-1532, Nov. 2007.

[115] G. Manfredi. Non-relativistic limits of Maxwell's equations. European Journal of Physics, 34(4):859, 2013.

[116] R. J. Mason. Implicit moment particle simulation of plasmas. J. Comput. Phys., 41(2):233-244, 1981.

[117] R. J. Mason. An electromagnetic field algorithm for 2D implicit plasma simulation. J. Comput. Phys., 71(2):429-473, 1987.

[118] A. Mikhailovskii. Instabilities in a Confined Plasma. Taylor \& Francis, 1 edition, Jan. 1998. 
[119] K. Miyamoto. Plasma physics and controlled nuclear fusion. Springer, Berlin [etc.], 2005.

[120] F. M. Mohammed Lemou. Micro-macro schemes for kinetic equations including boundary layers. SIAM Journal on Scientific Computing, 2012.

[121] J. Narski and M. Ottaviani. Asymptotic Preserving scheme for strongly anisotropic parabolic equations for arbitrary anisotropy direction. Computer Physics Communications, 185(12):3189-3203, Dec. 2014.

[122] S. Noelle, G. Bispen, K. Arun, M. Lukáčová-Medvid’ová, and C. Munz. A Weakly Asymptotic Preserving Low Mach Number Scheme for the Euler Equations of Gas Dynamics. SIAM Journal on Scientific Computing, 36(6):B989-B1024, Jan. 2014.

[123] M. Ottaviani. An alternative approach to field-aligned coordinates for plasma turbulence simulations. Physics Letters A, 375(15):1677-1685, 2011.

[124] L. Pareschi and G. Russo. Implicit-Explicit Runge-Kutta Schemes and Applications to Hyperbolic Systems with Relaxation. Journal of Scientific Computing, 25(1):129-155, Oct. 2005.

[125] L. Pareschi and G. Russo. Efficient Asymptotic Preserving Deterministic methods for the Boltzmann Equation. In Models and Computational Methods for Rarefied Flows, volume RTO-EN-AVT-194 of Lecture Series held at the von Karman Institute, pages (8-1)-(8-76). Rhode St. Genèse, Belgium, 2011.

[126] D. E. Post and R. Behrisch, editors. Physics of Plasma-Wall Interactions in Controlled Fusion. Springer US, Boston, MA, 1986.

[127] P. Ricci, F. D. Halpern, S. Jolliet, J. Loizu, A. Mosetto, A. Fasoli, I. Furno, and C. Theiler. Simulation of plasma turbulence in scrape-off layer conditions: the GBS code, simulation results and code validation. Plasma Physics and Controlled Fusion, 54(12):124047, 2012.

[128] P. Ricci, G. Lapenta, and J. U. Brackbill. A simplified implicit Maxwell solver. J. Comput. Phys., 183(1):117-141, 2002.

[129] P. H. Rutherford. Nonlinear growth of the tearing mode. Physics of Fluids (1958-1988), 16(11):1903-1908, Nov. 1973.

[130] D. D. Schnack. Lectures in Magnetohydrodynamics, volume 780 of Lecture Notes in Physics. Springer Berlin Heidelberg, Berlin, Heidelberg, 2009.

[131] B. Scott. Shifted metric procedure for flux tube treatments of toroidal geometry: Avoiding grid deformation. Physics of Plasmas, 8(2):447, 2001.

[132] B. D. Scott. Free-energy conservation in local gyrofluid models. Physics of Plasmas (1994-present), 12(10):102307, Oct. 2005. 
[133] B. W. Shanahan and B. D. Dudson. X-point modelling in linear configurations using BOUT ++ . Journal of Physics: Conference Series, 561:012015, Nov. 2014.

[134] P. Sharma and G. W. Hammett. Preserving monotonicity in anisotropic diffusion. Journal of Computational Physics, 227(1):123-142, Nov. 2007.

[135] K.-H. Spatschek. High temperature plasmas: theory and mathematical tools for laser and fusion plasmas. Wiley-VCH, Weinheim, 2012.

[136] W. M. Stacey. Fusion Plasma Physics. Wiley-VCH, Dec. 2005.

[137] P. C. Stangeby. The plasma boundary of magnetic fusion devices. Plasma physics series. Institute of Physics Pub, Bristol ; Philadelphia, 2000.

[138] A. Taflove and S. C. Hagness. Computational electrodynamics: the finitedifference time-domain method. Artech House antennas and propagation library. Artech House, Boston, 3rd ed edition, 2005.

[139] P. Tamain, P. Ghendrih, E. Tsitrone, V. Grandgirard, X. Garbet, Y. Sarazin, E. Serre, G. Ciraolo, and G. Chiavassa. TOKAM-3d: A 3d fluid code for transport and turbulence in the edge plasma of Tokamaks. Journal of Computational Physics, 229(2):361-378, Jan. 2010.

[140] M. Tang. Second order all speed method for the isentropic Euler equations. Kinetic and Related Models, 5(1):155-184, Jan. 2012.

[141] L. Tonks and I. Langmuir. A general theory of the plasma of an arc. Physical Review, 34(6):876-922, Sept. 1929.

[142] C. Tronci and E. Camporeale. Neutral Vlasov kinetic theory of magnetized plasmas. Physics of Plasmas (1994-present), 22(2):020704, Feb. 2015.

[143] C. Tronci, E. Tassi, E. Camporeale, and P. J. Morrison. Hybrid VlasovMHD models: Hamiltonian vs. non-Hamiltonian. Plasma Physics and Controlled Fusion, 56(9):095008, Sept. 2014. arXiv: 1403.2773.

[144] G. Tóth, B. van der Holst, I. V. Sokolov, D. L. De Zeeuw, T. I. Gombosi, F. Fang, W. B. Manchester, X. Meng, D. Najib, K. G. Powell, Q. F. Stout, A. Glocer, Y.-J. Ma, and M. Opher. Adaptive numerical algorithms in space weather modeling. Journal of Computational Physics, 231(3):870903, 2012.

[145] J. M. Wallace, J. U. Brackbill, and D. W. Forslund. An implicit moment electromagnetic plasma simulation in cylindrical coordinates. Journal of Computational Physics, 63(2):434 - 457, 1986.

[146] J. Wesson. Finite resistivity instabilities of a sheet pinch. Nuclear Fusion, 6(2):130-134, June 1966. 
[147] D. Winske and M. Hesse. Hybrid modeling of magnetic reconnection in space plasmas. Physica D: Nonlinear Phenomena, 77(1-3):268-275, 1994.

[148] D. Winske and N. Omidi. Hybrid codes: Methods and applications. Jan. 1991.

[149] D. Winske, L. Yin, N. Omidi, H. Karimabadi, and K. Quest. Hybrid Simulation Codes: Past, Present and Future-A Tutorial. In J. Büchner, M. Scholer, and C. T. Dum, editors, Space Plasma Simulation, volume 615, pages 136-165. Springer Berlin Heidelberg, Berlin, Heidelberg, 2003. 